\title{
ABSENCE OF EIGENVALUES OF ANALYTIC QUASI-PERIODIC SCHRÖDINGER OPERATORS ON $\mathbb{R}^{d}$
}

\author{
YUNFENG SHI
}

\begin{abstract}
In this paper we study on $L^{2}\left(\mathbb{R}^{d}\right)$ the quasi-periodic Schrödinger operator $H=-\Delta+\lambda V(x)$, where $V$ is a real analytic quasi-periodic function and $\lambda>0$. We first show that $H$ has no eigenvalues in low energy region. We also provide in low energy region the new phase transition parameter, i.e. the competition between the strength of coupling and the length for frequencies.
\end{abstract}

\section{INTRODUCTION AND MAIN RESULTS}

Let $H=-\Delta+V(x)$ be the Schrödinger operator defined on $L^{2}\left(\mathbb{R}^{d}\right)$. The question of determining for which potential $V(x)$ such $H$ has no positive eigenvalues attracted a great deal of attention over years, see e.g., [Kat59, Sim69, Agm70, FH83, JK85, IJ03, Mar19]. In general, those works require $V(x)$ to obey certain decaying law at $\infty$. This may exclude a large class of potentials without decaying, such as the quasi-periodic one. The aim of the present paper tries to establish absence of positive eigenvalues for some quasi-periodic Schrödinger operators on $\mathbb{R}^{d}$ for arbitrary $d \geq 1$.

Indeed, the quasi-periodic Schrödinger operator has important applications in condensed matter physics, and has been extensively studied in recent years, in particular in its discrete case (see [MJ17, Dam17] and references therein).

Considerable research results have been obtained for quasi-periodic Schrödinger operators on $\mathbb{R}$. In $1 D$ and periodic potentials case, the Floquet theory works well, and as a result the spectrum consists of intervals (bands) and is purely absolutely continuous $(a c)$. When the periodic potential is replaced by a quasi-periodic one, the spectral properties change dramatically resulting from the so-called smalldivisors effect. Dinaburg-Sinai [DS75] first proved the existence of ac spectrum by a using a KAM reducibility argument. This result was further developed later by Rüssmann [Rüs80] and Moser-Pöschel [MP84]. Surace [Sur90] showed for potentials of the form

$$
V(x)=\lambda(\cos x+\cos (\theta+\omega x)),
$$

the whole spectrum contains no eigenvalues if $\lambda$ is small and the frequency $\omega$ is Diophantine. He extended the multi-scale analysis (MSA) of [FS83, FSW90] to work in momentum space. The breakthrough came from Eliasson [Eli92], in which he proved for any nonconstant analytic quasi-periodic potential and Diophantine frequency, the spectrum is purely $a c$ in high energy region. His result also applied to the whole spectrum for small quasi-periodic potentials. Moreover, he showed

Date: March 30, 2021.

Key words and phrases. Absence of eigenvalues, Multi-dimensional continuous Schrödinger operators, Quasi-periodic potentials, Phase transition. 
the spectrum is a Cantor set for a generic set of analytic quasi-periodic potentials, which exhibits completely different spectral features compared with periodic potentials. For quasi-periodic potentials $\lambda V(x)$ with large $\lambda>0$, Anderson localization (AL, i.e., pure point spectrum with exponentially decaying eigenfunctions) and delocalization transition [YZ14] may be expected. Sorets-Spencer [SS91] improved Herman's subharmonic trick to obtain the positivity of Lyapunov exponent at low energy for potentials (1.1) if $\lambda \gg 1$. Fröhlich-Spencer-Wittwer [FSW90] proved the first AL for potentials of the form (1.1) at low energy if $\lambda \gg 1$. They performed a MSA for Green's functions in $x \in \mathbb{R}$ space directly. It has been proven by You-Zhou [YZ14] that there exists the phase transition from singular to purely ac spectrum for $1 D$ and two frequencies quasi-periodic operators if the coupling is large. Very recently, Binder-Kinzebulatov-Voda [BKV17] proved a non-perturbative AL (i.e. AL under only positive Lyapunov exponents assumption) for general analytic potentials in finite energy intervals by applying methods of [BG00, GS08]. For more results in $1 D$ quasi-periodic setting, we refer to [Bje06, DG14, Liu18].

If one increases the space dimension to $d \geq 2$, the situation becomes significantly more complicated and much less results were obtained in this setting. Unlike the $1 D$ case, it was conjectured (i.e. the Bethe-Sommerfeld conjecture [Par08, PS10]) that the spectrum of any periodic Schrödinger operator in higher dimensions contains finitely many gaps. By contrast, Damanik-Fillman-Gorodetski [DFG19] had constructed $d D$ almost-periodic Schrödinger operators whose spectrum is a generalized Cantor set of zero Lebesgue measure. The first result concerning ac spectrum of a $2 D$ quasi-periodic Schrödinger operator was due to Karpeshina-Shterenberg [KS19], where they obtained the existence of ac spectrum at high energy, and the spectrum contains a semi-axis. The proof of this elegant result is based on their new MSA in momentum space. Before this work, they [KS13] got a similar result for $(-\Delta)^{l}+V(x)$ with $l \geq 2$. We also mention the work of Karpeshina-LeeShterenberg-Stolz [KLSS17], in which the existence of ballistic transport for the Schrödinger operator with limit-periodic or quasi-periodic potential in dimension two was established. There are also some results [PS16, PS12] for quasi-periodic (even almost periodic) operators on $\mathbb{R}^{d}$ in dealing with asymptotic expansion of the spectral functions (such as the IDS).

If $d \geq 3$, as far as we know, there are no localization or de-localization results available for a quasi-periodic Schrödinger operator on $\mathbb{R}^{d}$. This is the other motivation of the present work.

In this paper we study on $\mathbb{R}^{d}(d \geq 1)$ the Schrödinger operators with analytic quasi-periodic potentials $\lambda V(x)$ and prove the absence of eigenvalues in low energy region. Combined our main theorem and results of [Bje06, Bje07, YZ14, BKV17], we also provide the new phase transition parameter in low energy region (see (4) of Remark 1.1 in the following).

Here is the set up of our main result:

Let $b_{i} \in \mathbb{N}(1 \leq i \leq d)$ and $b=\sum_{i=1}^{d} b_{i}>d$. Define $\mathbb{T}^{b}=\prod_{i=1}^{d}(\mathbb{R} / 2 \pi \mathbb{Z})^{b_{i}}$. Write $\vec{\theta}=\left(\vec{\theta}_{1}, \cdots, \vec{\theta}_{d}\right) \in \mathbb{T}^{b}$ for $\vec{\theta}_{i}=\left(\theta_{i 1}, \cdots, \theta_{i b_{i}}\right) \in \mathbb{T}^{b_{i}}(1 \leq i \leq d)$. Similarly, let $\vec{\omega}=$ $\left(\vec{\omega}_{1}, \cdots, \vec{\omega}_{d}\right) \in[0,2 \pi]^{b}$ with $\vec{\omega}_{i} \in[0,2 \pi]^{b_{i}}(1 \leq i \leq d)$. Let $x=\left(x_{1}, \cdots, x_{d}\right) \in \mathbb{R}^{d}$ and define $x \vec{\omega}=\left(x_{1} \vec{\omega}_{1}, \cdots, x_{d} \vec{\omega}_{d}\right) \in \mathbb{R}^{b}$.

We consider on $L^{2}\left(\mathbb{R}^{d}\right)$ the following Schrödinger operators with quasi-periodic potentials

$$
H(\vec{\theta})=-\Delta+\lambda V(\vec{\theta}+K x \vec{\omega})
$$


where the real function $V$ is the potential, $\lambda \geq 0$ is the coupling, $\vec{\omega}$ is the frequency, $\vec{\theta}$ is the phase and $K>0$.

We focus on the analytic function $V \in C^{\omega}\left(\mathbb{T}^{b} ; \mathbb{R}\right)$ satisfying $\int_{\mathbb{T}^{b}} V(\vec{\theta}) \mathrm{d} \vec{\theta}=0$. Without loss of generality, we may assume for some $\rho>0$ and $\forall \mathbf{k}=\left(\mathbf{k}_{1}, \cdots, \mathbf{k}_{d}\right) \in$ $\mathbb{Z}^{b}\left(\mathbf{k}_{i}=\left(k_{i 1}, \cdots, k_{i b_{i}}\right) \in \mathbb{Z}^{b_{i}}, 1 \leq i \leq d\right)$

$$
\left|\widehat{V}_{\mathbf{k}}\right| \leq e^{-\rho|\mathbf{k}|}
$$

where $\widehat{V}_{\mathbf{k}}=\int_{\mathbb{T}^{b}} V(\vec{\theta}) e^{-\mathbf{i k} \cdot \vec{\theta}} \mathrm{d} \vec{\theta}$ (with $\mathbf{k} \cdot \vec{\theta}=\sum_{i=1}^{d} \mathbf{k}_{i} \cdot \vec{\theta}_{i}=\sum_{i=1}^{d} \sum_{j=1}^{b_{i}} k_{i j} \theta_{i j}$ ) is the Fourier coefficient of $V(\vec{\theta})$ and $|\mathbf{k}|=\max _{1 \leq i \leq d, 1 \leq j \leq b_{i}}\left|k_{i j}\right|$.

Denote by mes(.) the Lebesgue measure. We have

Theorem 1.1. Let $H(\vec{\theta})$ be defined by (1.2) with $V$ satisfying (1.3). Then there is $c_{\star}=c_{\star}(b, d)>0$ (depending on $b_{1}, \cdots, b_{d}, d$ ) such that, for $\delta>0$ and $0<c_{1}<c_{\star}$, there exists $\varepsilon_{0}=\varepsilon_{0}\left(\delta, c_{1}, \rho, b, d\right)>0$ such that the following holds: If $0<\frac{\lambda}{K^{2}} \leq \varepsilon_{0}$, then there is some $\Omega=\Omega(K, \lambda) \subset[0,2 \pi]^{b}$ satisfying $\operatorname{mes}\left([0,2 \pi]^{b} \backslash \Omega\right) \leq \delta$ such that $H(\vec{\theta})$ has no eigenvalues in $\left[-K^{2}\left(\log \frac{K^{2}}{\lambda}\right)^{\frac{1}{2 c_{1}}}, K^{2}\left(\log \frac{K^{2}}{\lambda}\right)^{\frac{1}{2 c_{1}}}\right]$ for every $\vec{\theta} \in \mathbb{T}^{b}$ and $\vec{\omega} \in \Omega$.

Remark 1.1. (1) Theorem 1.1 is non-vacuous, i.e., there exists a portion of the spectrum in $\mathcal{I}=\left[-K^{2}\left(\log \frac{K^{2}}{\lambda}\right)^{\frac{1}{2 c_{1}}}, K^{2}\left(\log \frac{K^{2}}{\lambda}\right)^{\frac{1}{2 c_{1}}}\right]$. Indeed (see Lemma A.1 in the Appendix), one can show for any $E \geq 0$ and $M \geq \lambda|V|_{\max }$, $\sigma(H(\vec{\theta})) \cap[E-M, E+M] \neq \emptyset$, where $\sigma(\cdot)$ denotes the spectrum. In particular, $\sigma(H(\vec{\theta})) \cap[-M, M] \neq \emptyset$ for any $M>\lambda|V|_{\max }$. Moreover, it is obvious that $\mathcal{I} \nearrow \mathbb{R}$ as $\lambda \rightarrow 0$ or $K \rightarrow \infty$.

(2) We make no smallness assumptions on $\lambda>0$, and prove the first purely continuous spectrum result in low energy region for quasi-periodic operators on $\mathbb{R}^{d}$ for arbitrary $d \geq 1$. This can be regarded as a supplement of results of [KS19], where the existence of $a c$ spectrum for a $2 D$ quasi-periodic Schrödinger operator was established in high energy region.

(3) The theorem suggests that the length of frequencies is relevant for the spectral properties of continuous quasi-periodic Schrödinger operators.

(4) If $d=1, b_{1}>1$ and $V$ satisfies some conditions, it was proved in [BKV17] (combined with results of [Bje06]) that for $\lambda \geq \lambda_{0}\left(K \vec{\omega}, b_{1}\right)>0$ the spectrum in $\left[0, \lambda^{2 / 3}\right]$ is pure point with exponential decay eigenfunctions (i.e. $\mathrm{AL}$ ) for a.e. Diophantine $\vec{\omega}$. By contrast, our Theorem 1.1 implies that there are no eigenvalues in $[0, C \lambda]$ at all for any $\lambda>0$ if $K \geq C \sqrt{\lambda}$. Those motivate us to provide the new phase transition parameter $\frac{\log K}{\log \lambda}$ from pure point to purely continuous spectrum in low energy region. Precisely, there may exist a competition between $\lambda$ and $K: \frac{\log K}{\log \lambda}>C>0$ implies purely continuous spectrum; and $\frac{\log K}{\log \lambda} \leq C$ may show pure point spectrum (even $\mathrm{AL}$ ). We refer to [YZ14] for the phase transition parameter involving the coupling and energy: $\frac{\log E}{\log \lambda}$.

(5) Our results can be extended to the case $H=(-\Delta)^{l}+\lambda V(\vec{\theta}+K x \vec{\omega})$, where $l \in \mathbb{Z}, l \geq 1$ and $V(\cdot)$ is Gevrey regular (see [Shi19b]). 
(6) It should be true that the spectrum in $\mathcal{I}$ is purely absolutely continuous for $0<\frac{\lambda}{K^{2}} \ll 1$ and for most $\vec{\omega}$. However, we think Anderson localization occurs at low energy for $\frac{\lambda}{K^{2}} \gg 1$ and for most $\vec{\omega}$.

We outline the proof of Theorem 1.1:

The main scheme is to perform MSA in momentum space $\Theta \in \mathbb{R}^{d}$. Let $E$ be an eigenvalue of $H(\vec{\theta})$, i.e. $H(\vec{\theta}) \Psi=E \Psi$ for some $0 \neq \Psi \in L^{2}\left(\mathbb{R}^{d}\right)$. Let

$$
\mathbf{k} \vec{\omega}=\left(\mathbf{k}_{1} \cdot \vec{\omega}_{1}, \cdots, \mathbf{k}_{d} \cdot \vec{\omega}_{d}\right) .
$$

Denote by $\widehat{\Psi}$ the Fourier transformation of $\Psi$. Then by direct computations (see Lemma 4.1 for details), the vector

$$
Z=\left\{Z_{\mathbf{k}}=e^{-\mathbf{i k} \cdot \vec{\theta}} \widehat{\Psi}\left(\Theta^{\prime}+K \mathbf{k} \vec{\omega}\right)\right\}_{\mathbf{k} \in \mathbb{Z}^{b}}
$$

will satisfy $h(\Theta) Z=\frac{E}{K^{2}} Z$, where

$$
(h(\Theta) Z)_{\mathbf{k}}=\sum_{\mathbf{k}^{\prime} \in \mathbb{Z}^{b}} \varepsilon \widehat{V}_{\mathbf{k}-\mathbf{k}^{\prime}} Z_{\mathbf{k}^{\prime}}+\sum_{i=1}^{d}\left(\Theta_{i}+\mathbf{k}_{i} \cdot \vec{\omega}_{i}\right)^{2} Z_{\mathbf{k}}, \varepsilon=\frac{\lambda}{K^{2}}, \Theta=\frac{\Theta^{\prime}}{K} .
$$

In addition, we can even show $Z_{\mathbf{k}}$ grows at most polynomially in $\mathbf{k}$ for a.e. $\Theta$. Thus the asymptotic property of $Z_{\mathbf{k}}$ can be controlled by fast off-diagonal decaying properties of the Green's functions (if exist)

$$
G_{\Lambda}(E ; \Theta)=\left(R_{\Lambda}(h(\Theta)-E) R_{\Lambda}\right)^{-1}, \Lambda \subset \mathbb{Z}^{b} .
$$

At this stage, it suffices to get certain off-diagonal decaying estimates on $G_{\Lambda}(E ; \Theta)$ as $|\Lambda| \rightarrow \infty$. For this purpose, it needs to make quantitative restrictions on $\vec{\omega}, \Theta$ and even on $E$ due to the small-divisors difficulty. Usually, the KAM and MSA methods are powerful to overcome the small-divisors. In continuous quasi-periodic operators case, Surace [Sur90] performed the first MSA scheme ${ }^{1}$ in momentum space $\Theta \in \mathbb{R}$ but restricted to diagonal part of the form

$$
\left(\Theta+k_{1}+k_{2} \omega\right)^{2},\left(k_{1}, k_{2}\right) \in \mathbb{Z}^{2} .
$$

Because of this special structure, Surace can even obtain uniform in $E \in \mathbb{R}$ estimate. However, the proof of [Sur90] depends heavily on eigenvalue variations and is hard to work for $\Theta$ in higher dimensions. For recent $\Theta \in \mathbb{R}$ type MSA, we also refer to [DG14] in dealing with the inverse spectral theory for quasi-periodic operators on $\mathbb{R}$.

For general $\Theta \in \mathbb{R}^{d}$ in momentum space the only known MSA is developed in recent papers [KS13, KS19] for $d=2$. However, for arbitrary $d \geq 1$ similar type of questions has been extensively studied in case $\Theta \in \mathbb{T}^{d}$ [BGS02, Bou07, BK19, JLS20, Shi19b] via techniques of semi-algebraic geometry arguments and subharmonic estimates. As compared with compact momentum case (i.e. $\Theta \in \mathbb{T}^{d}$ ), there comes new difficulty for general $\Theta \in \mathbb{R}^{d}$ : For any finite $\Lambda \subset \mathbb{Z}^{b}$,

$$
\sup _{\mathbf{k} \in \Lambda}\left|\sum_{i=1}^{d}\left(\Theta_{i}+\mathbf{k}_{i} \cdot \vec{\omega}_{i}\right)^{2}-E\right| \ll 1
$$

\footnotetext{
${ }^{1}$ The main scheme is similar that of [FSW90].
} 
may frequently occur while $|\Theta| \gg 1$ and $|E| \gg 1$. To avoid this difficulty, one may make the assumption that for some $C>0{ }^{2}$,

$$
-C \leq E \leq C .
$$

Once (1.5) is satisfied, $\Theta$ stays essentially in a compact set of size $\sim|\Lambda|$, and the small divisors presented in (1.4) could be handled via methods of [Bou07, JLS20]. The elimination frequencies argument of Bourgain [Bou07] based on semi-algebraic geometry theory is likely applicable. Consequently, we may expect that $H(\vec{\theta})$ has no eigenvalues in interval $\left[-C K^{2}, C K^{2}\right]$ if $0<\varepsilon \leq \varepsilon_{0}(C)$.

It is reasonable that the absence of eigenvalues for $H(\vec{\theta})$ should hold in a much longer energy interval, which of course needs a proof with new ideas. In this paper we will provide such a proof based on the following observations:

- The first step of MSA is in general based on perturbative argument, in particular the Neumann series expansions. It is important that the large deviation estimate (LDE) for Green's functions is valid for every $E \in \mathbb{R}$ in this step.

- Once the first step of MSA is finished, we will deal with the second iteration step. To propagate the LDE, some restrictions on $\vec{\omega}$ are necessary, which is essentially necessary even in $\Theta \in \mathbb{T}^{d}$ case. What's new here is that we can make quantitative restrictions on $E$ in this step. More precisely, the first step MSA implies that LDE holds in scales interval $N_{0} \leq N \leq|\log \varepsilon|^{C}$. The second MSA step will start with $N=|\log \varepsilon|^{C}$. To avoid (1.4), we can actually set $|E| \leq|\log \varepsilon|^{C}$. In this energy interval, we could perform the iteration similar to that of [Bou07, JLS20] together with some technical improvements.

- Since $|E| \leq|\log \varepsilon|^{C}$ is negligible as compared with the later general MSA scales $N \gg|\log \varepsilon|^{C}$, the iterations become similar to that done in the second step.

In conclusion, our new aspect here is that we focus on the MSA (in the momentum space $\left.\Theta \in \mathbb{R}^{d}\right)$ in the first two steps and make effective restrictions on $E$ in the second step.

Of course, the final aim is to show $H(\vec{\theta})$ has no eigenvalues on $\mathbb{R}$, which seems difficult to handle via the present method.

The structure of the paper is as follows. Some preliminaries are introduced in $\S 2$. The MSA in momentum space is established in $\S 3$. In $\S 4$, we finish the proof of Theorem 1.1. Some useful estimates are included in the Appendix.

\section{Preliminaries}

2.1. Some notation. For any $x \in \mathbb{R}^{d}$, let $|x|=\max _{1 \leq i \leq d}\left|x_{i}\right|$. For $\Lambda \subset \mathbb{R}^{d}$, we introduce

$$
\operatorname{diam}(\Lambda)=\sup _{n, n^{\prime} \in \Lambda}\left|n-n^{\prime}\right|, \operatorname{dist}(m, \Lambda)=\inf _{n \in \Lambda}|m-n| .
$$

For $\Theta \in \mathbb{R}^{d}$ and $1 \leq j \leq d$, let $\Theta_{j}^{\urcorner}=\left(\Theta_{1}, \cdots, \Theta_{j-1}, \Theta_{j+1} \cdots, \Theta_{d}\right) \in \mathbb{R}^{d-1}$.

For $x \in \mathbb{R}^{d_{1}}$ and $\emptyset \neq X \subset \mathbb{R}^{d_{1}+d_{2}}$, define the $x$-section of $X$ to be

$$
X(x)=\left\{y \in \mathbb{R}^{d_{2}}:(x, y) \in X\right\} .
$$

\footnotetext{
${ }^{2}$ This assumption is essentially satisfied in [BGS02, Bou07, BK19, JLS20, Shi19b].
} 
For example, $X\left(\Theta_{j}\right)=\left\{\Theta_{j} \in \mathbb{R}:\left(\Theta_{j}, \Theta_{j}^{\urcorner}\right) \in X\right\}$ if $\emptyset \neq X \subset \mathbb{R}^{d}$.

Throughout this paper, we assume $\rho \in(0,1)$ for simplicity.

2.2. Aubry duality. It is easy to check that if

$$
\Psi(x)=e^{\mathbf{i} \Theta \cdot x} \sum_{\mathbf{k} \in \mathbb{Z}^{b}} \Psi_{\mathbf{k}} e^{\mathbf{i k} \cdot(\vec{\theta}+x \vec{\omega})}
$$

is a solution of $(-\Delta+\varepsilon V(\vec{\theta}+x \vec{\omega})) \Psi(x)=E \Psi(x)$ (i.e. the Floquet-Bloch solution), then $\left\{\Psi_{\mathbf{k}}\right\}_{\mathbf{k} \in \mathbb{Z}^{b}}$ satisfies the following equation

$$
\sum_{\mathbf{k}^{\prime} \in \mathbb{Z}^{b}} \varepsilon \widehat{V}_{\mathbf{k}-\mathbf{k}^{\prime}} \Psi_{\mathbf{k}^{\prime}}+\sum_{i=1}^{d}\left(\Theta_{i}+\mathbf{k}_{i} \cdot \vec{\omega}_{i}\right)^{2} \Psi_{\mathbf{k}}=E \Psi_{\mathbf{k}} .
$$

This motivates us to study the following unbounded quasi-periodic operators on $\mathbb{Z}^{b}$

$$
(h(\Theta) Z)_{\mathbf{k}}=\sum_{\mathbf{k}^{\prime} \in \mathbb{Z}^{b}} \varepsilon \widehat{V}_{\mathbf{k}-\mathbf{k}^{\prime}} Z_{\mathbf{k}^{\prime}}+\sum_{i=1}^{d}\left(\Theta_{i}+\mathbf{k}_{i} \cdot \vec{\omega}_{i}\right)^{2} Z_{\mathbf{k}} .
$$

We call $h(\Theta)$ the Aubry duality of $-\Delta+\varepsilon V(\vec{\theta}+x \vec{\omega})$. For Aubry duality results in discrete case, we refer to [BJ02, JK16, Shi19b].

2.3. Green's functions and elementary regions. If $\Lambda \subset \mathbb{Z}^{b}$, denote $h_{\Lambda}(\Theta)=$ $R_{\Lambda} h(\Theta) R_{\Lambda}$, where $R_{\Lambda}$ is the restriction operator and $h(\Theta)$ is given by (2.1). Define the Green's function as

$$
G_{\Lambda}(E ; \Theta)=\left(h_{\Lambda}(\Theta)-E+\mathbf{i} 0\right)^{-1} .
$$

Denote by $Q_{N}$ an elementary region of size $N$ centered at 0 (as in [JLS20]):

$$
Q_{N}=[-N, N]^{b} \text { or } Q_{N}=[-N, N]^{b} \backslash\left\{\mathbf{k} \in \mathbb{Z}^{b}: k_{i j} \in \varsigma_{i j}, 1 \leq i \leq d, 1 \leq j \leq b_{i}\right\},
$$

where $\varsigma_{i j} \in\{\{n<0\},\{n>0\}, \emptyset\}$ and at least two $\varsigma_{i j}$ are not $\emptyset$. Denote by $\mathcal{E}_{N}^{0}$ the set of all elementary regions of size $N$ centered at 0 . Let $\mathcal{E}_{N}$ be the set of all translates of elementary regions: $\mathcal{E}_{N}=\bigcup_{\mathbf{k} \in \mathbb{Z}^{b}, Q_{N} \in \mathcal{E}_{N}^{0}}\left\{\mathbf{k}+Q_{N}\right\}$.

\subsection{Semi-algebraic sets.}

Definition 2.1 (Chapter 9, [Bou05]). A set $\mathcal{S} \subset \mathbb{R}^{n}$ is called a semi-algebraic set if it is a finite union of sets defined by a finite number of polynomial equalities and inequalities. More precisely, let $\left\{P_{1}, \cdots, P_{s}\right\} \subset \mathbb{R}\left[x_{1}, \cdots, x_{n}\right]$ be a family of real polynomials whose degrees are bounded by $d$. A (closed) semi-algebraic set $\mathcal{S}$ is given by an expression

$$
\mathcal{S}=\bigcup_{j} \bigcap_{\ell \in \mathcal{L}_{j}}\left\{x \in \mathbb{R}^{n}: P_{\ell}(x) \varsigma_{j \ell} 0\right\},
$$

where $\mathcal{L}_{j} \subset\{1, \cdots, s\}$ and $\varsigma_{j \ell} \in\{\geq, \leq,=\}$. Then we say that $\mathcal{S}$ has degree at most $s d$. In fact, the degree of $\mathcal{S}$ which is denoted by $\operatorname{deg}(\mathcal{S})$, means the smallest $s d$ over all representations as in (2.2).

Lemma 2.2 (Tarski-Seidenberg Principle, [Bou05]). Denote by $(x, y) \in \mathbb{R}^{d_{1}+d_{2}}$ the product variable. If $\mathcal{S} \subset \mathbb{R}^{d_{1}+d_{2}}$ is semi-algebraic of degree $B$, then its projections $\operatorname{Proj}_{x} \mathcal{S} \subset \mathbb{R}^{d_{1}}$ and $\operatorname{Proj}_{y} \mathcal{S} \subset \mathbb{R}^{d_{2}}$ are semi-algebraic of degree at most $B^{C}$, where $C=C\left(d_{1}, d_{2}\right)>0$. 
Lemma 2.3 ([Bou07]). Let $\mathcal{S} \subset[0,1]^{d=d_{1}+d_{2}}$ be a semi-algebraic set of degree $\operatorname{deg}(\mathcal{S})=B$ and $\operatorname{mes}_{d}(\mathcal{S}) \leq \eta$, where $\log B \ll \log \frac{1}{\eta}$.

Denote by $\left(x_{1}, x_{2}\right) \in[0,1]^{d_{1}} \times[0,1]^{d_{2}}$ the product variable. Suppose $\eta^{\frac{1}{d}} \leq \epsilon$. Then there is a decomposition of $\mathcal{S}$ as

$$
\mathcal{S}=\mathcal{S}_{1} \cup \mathcal{S}_{2}
$$

with the following properties. The projection of $\mathcal{S}_{1}$ on $[0,1]^{d_{1}}$ has small measure

$$
\operatorname{mes}_{d_{1}}\left(\operatorname{Proj}_{x_{1}} \mathcal{S}_{1}\right) \leq B^{C(d)} \epsilon
$$

and $\mathcal{S}_{2}$ has the transversality property

$$
\operatorname{mes}_{d_{2}}\left(\mathcal{L} \cap \mathcal{S}_{2}\right) \leq B^{C(d)} \epsilon^{-1} \eta^{\frac{1}{d}}
$$

where $\mathcal{L}$ is any $d_{2}$-dimensional hyperplane in $\mathbb{R}^{d}$ s.t., $\max _{1 \leq j \leq d_{1}}\left|\operatorname{Proj}_{\mathcal{L}}\left(e_{j}\right)\right|<\epsilon$, where we denote by $e_{1}, \cdots, e_{d_{1}}$ the $x_{1}$-coordinate vectors.

\section{LDT FOR GREEN's FUnCTIONS}

The main result of this section is the following LDT for Green's functions.

Theorem 3.1 (LDT). There exists $c_{\star}(b, d)>0$ such that the following holds: For any $0<c_{1} \leq c_{\star}$, there exists $N_{0}=N_{0}\left(c_{1}, \rho, b, d\right)>0$ such that if

$$
\log \log \frac{1}{\varepsilon} \geq N_{0},
$$

then for all $N \geq N_{0}$ we have

(i) There is some semi-algebraic set $\Omega_{N}=\Omega_{N}\left(c_{1}, \varepsilon, \rho, b, d\right) \subset[0,2 \pi]^{b}$ with $\operatorname{deg}\left(\Omega_{N}\right) \leq N^{4 d}$, and as $\varepsilon \rightarrow 0$,

$$
\operatorname{mes}\left([0,2 \pi]^{b} \backslash \bigcap_{N \geq N_{0}} \Omega_{N}\right) \rightarrow 0 .
$$

(ii) If $\vec{\omega} \in \Omega_{N}$ and $E \in \mathbb{R}$ satisfying $|E| \leq|\log \varepsilon|^{\frac{1}{2 c_{1}}}$, then there exists some set $\mathbf{X}_{N}=\mathbf{X}_{N}(\vec{\omega}, E) \subset \mathbb{R}^{d}$ such that

$$
\sup _{1 \leq j \leq d, \Theta_{j}^{\neg} \in \mathbb{R}^{d-1}} \operatorname{mes}\left(\mathbf{X}_{N}\left(\Theta_{j}^{\urcorner}\right)\right) \leq e^{-N^{c_{1}}}
$$

and for $\Theta \notin \mathbf{X}_{N}, Q \in \mathcal{E}_{N}^{0}$,

$$
\begin{aligned}
\left\|G_{Q}(E ; \Theta)\right\| & \leq e^{\sqrt{N}}, \\
\left|G_{Q}(E ; \Theta)\left(\mathbf{n}, \mathbf{n}^{\prime}\right)\right| & \leq e^{-\frac{\rho}{10}\left|\mathbf{n}-\mathbf{n}^{\prime}\right|} \text { for }\left|\mathbf{n}-\mathbf{n}^{\prime}\right| \geq N / 10 .
\end{aligned}
$$

Proof of Theorem 3.1. The proof is based on the multi-scale analysis scheme as in [Bou07, JLS20]. However, we formulate it into three steps.

STEP 1: The First Step

In this initial step we use a perturbative argument. It is important that in this step we obtain uniform measure estimates both on $E \in \mathbb{R}$ and $\vec{\omega}$.

Lemma 3.2. Given $\delta>0$, let

$$
\mathbf{X}_{N}=\bigcup_{|\mathbf{k}| \leq N}\left\{\Theta \in \mathbb{R}^{d}:\left|\sum_{i=1}^{d}\left(\Theta_{i}+\mathbf{k}_{i} \cdot \vec{\omega}_{i}\right)^{2}-E\right|<\delta\right\}
$$


Then

$$
\sup _{1 \leq j \leq d, \Theta_{j}^{\urcorner} \in \mathbb{R}^{d-1}} \operatorname{mes}\left(\mathbf{X}_{N}\left(\Theta_{j}^{\urcorner}\right)\right) \leq C(2 N+1)^{b} \sqrt{\delta},
$$

where $C>0$ is an absolute constant.

Moreover, if

$$
\varepsilon^{-1} \geq 2 \delta^{-1}(2 N+1)^{b}
$$

then for any $\Theta \notin \mathbf{X}_{N}$ and $\Lambda \subset[-N, N]^{b}$,

$$
\begin{aligned}
\left\|G_{\Lambda}(E ; \Theta)\right\| & \leq 2 \delta^{-1} \\
\left|G_{\Lambda}(E ; \Theta)\left(\mathbf{n}, \mathbf{n}^{\prime}\right)\right| & \leq 2 \delta^{-1} e^{-\rho\left|\mathbf{n}-\mathbf{n}^{\prime}\right|} .
\end{aligned}
$$

Proof. The proof is perturbative and based on the Neumann series argument: The proof of measure bound is based on Lemma 4.7 in [Shi19a], and the estimate on Green's functions can be found in the proof of Theorem 4.3 in [JLS20].

If we take $C(2 N+1)^{b} \sqrt{\delta}=e^{-N^{c_{1}}}$ in the above lemma, we obtain

Proposition 3.3. Let $0<c_{1}<1 / 4$. Then there exists $N_{0}=N_{0}\left(c_{1}, \rho, b, d\right)>0$ such that the following: If $N_{0} \leq N \leq|\log \varepsilon|^{\frac{1}{2 c_{1}}}$, then for all $E \in \mathbb{R}$ and $\vec{\omega} \in[0,2 \pi]^{b}$ there is some $\mathbf{X}_{N}=\mathbf{X}_{N}(E, \vec{\omega}) \subset \mathbb{R}^{d}$ such that

$$
\sup _{1 \leq j \leq d, \Theta_{j}^{\urcorner} \in \mathbb{R}^{d-1}} \operatorname{mes}\left(\mathbf{X}_{N}\left(\Theta_{j}^{\urcorner}\right)\right) \leq e^{-N^{c_{1}}},
$$

and, if $\Theta \notin \mathbf{X}_{N}$ and $Q \in \mathcal{E}_{N}^{0}$,

$$
\begin{aligned}
\left\|G_{Q}(E ; \Theta)\right\| & \leq e^{\sqrt{N}} \\
\left|G_{Q}(E ; \Theta)\left(\mathbf{n}, \mathbf{n}^{\prime}\right)\right| & \leq e^{-\frac{4 \rho}{5}\left|\mathbf{n}-\mathbf{n}^{\prime}\right|} \text { for }\left|\mathbf{n}-\mathbf{n}^{\prime}\right| \geq N / 10 .
\end{aligned}
$$

Proof. Let $\mathbf{X}_{N}$ be given by Lemma 3.2. Then the measure bound (3.1) can be derived from choosing $C(2 N+1)^{b} \sqrt{\delta}=e^{-N^{c_{1}}}$, i.e.,

$$
\delta=C^{-2}(2 N+1)^{-2 b} e^{-2 N^{c_{1}}} .
$$

We then turn to the Green's function estimates. Let $\Theta \notin \mathbf{X}_{N}$. Since (3.4), the condition $\varepsilon^{-1} \geq \delta^{-1}(2 N+1)^{b}$ is equivalent to

$$
\varepsilon^{-1} \geq 2 C^{2}(2 N+1)^{3 b} e^{2 N^{c_{1}}}
$$

It suffices to let $N \geq N_{0}\left(c_{1}, b, d\right)>0$ and

$$
\varepsilon^{-1} \geq e^{N^{2 c_{1}}} \geq 2 C^{2}(2 N+1)^{3 b} e^{2 N^{c_{1}}}
$$

that is $N_{0}\left(c_{1}, b, d\right) \leq N \leq|\log \varepsilon|^{\frac{1}{2 c_{1}}}$. From (3.5) and $0<c_{1}<1 / 4$, we have $2 \delta^{-1} \leq e^{N^{2 c_{1}}} \leq e^{\sqrt{N}}$, which implies (3.2). Finally, the exponential decay bound (3.3) follows from: If $\left|\mathbf{n}-\mathbf{n}^{\prime}\right| \geq N / 10$ and $N \geq N_{0}\left(c_{1}, \rho, b, d\right)>0$, then

$$
\left|G_{Q}(E ; \Theta)\left(\mathbf{n}, \mathbf{n}^{\prime}\right)\right| \leq e^{\sqrt{N}-\frac{\rho}{50} N} e^{-\frac{4 \rho}{5}\left|\mathbf{n}-\mathbf{n}^{\prime}\right|} \leq e^{-\frac{4 \rho}{5}\left|\mathbf{n}-\mathbf{n}^{\prime}\right|} .
$$




\section{STEP 2: The Second Step}

In the second step we will propagate LDT from (scales) $\left[N_{0},|\log \varepsilon|^{\frac{1}{2 c_{1}}}\right]$ to

$$
\left[|\log \varepsilon|^{\frac{1}{2 c_{1}}}, e^{|\log \varepsilon|^{1 / 4}}\right] .
$$

For this purpose, further removal of $\vec{\omega}$ is necessary and the semi-algebraic geometry arguments established by Bourgain [Bou07] play a key role. In what follows we always assume

$$
E \in \mathcal{I}_{\varepsilon}=\left[-|\log \varepsilon|^{\frac{1}{2 c_{1}}},\left.\log \varepsilon\right|^{\frac{1}{2 c_{1}}}\right] .
$$

Denote $\mathbf{B}(N)=\left\{\Theta \in \mathbb{R}^{d}:|\Theta| \leq N\right\}$. Then we have

Proposition 3.4. Let $|\log \varepsilon|^{\frac{1}{2 c_{1}}} \leq N \leq e^{|\log \varepsilon|^{1 / 4}}, N_{1} \sim(\log N)^{2 / c_{1}}$. Then there exist constants

$$
0<c_{2}(b, d)<c_{3}(b, d)<c_{4}(b, d) \ll 1,
$$

and semi-algebraic set $\Omega_{N} \subset[0,2 \pi]^{b}$ satisfying

$$
\operatorname{deg}\left(\Omega_{N}\right) \leq N^{4 d}, \operatorname{mes}\left([0,2 \pi]^{b} \backslash \Omega_{N}\right) \leq N^{-c_{2}}
$$

such that the following holds: If $\log \log \frac{1}{\varepsilon} \geq N_{0}\left(c_{1}, b, d\right)>0$ and $\vec{\omega} \in \Omega_{N}$, then for all $(E, \Theta) \in \mathcal{I}_{\varepsilon} \times \mathbf{B}\left(100 b N^{2}\right)$ there is some $\frac{1}{10} N^{c_{3}}<M<10 N^{c_{4}}$ such that for all $\mathbf{k} \in[-M, M]^{b} \backslash\left[-M^{\frac{1}{10 b}}, M^{\frac{1}{10 b}}\right]^{b}$, one has $\Theta+\mathbf{k} \vec{\omega} \notin \mathbf{X}_{N_{1}}$, here $\mathbf{X}_{N_{1}}$ is given by Proposition 3.3.

Remark 3.1. The conditions $\log \log \frac{1}{\varepsilon} \geq N_{0}\left(c_{1}, b, d\right)>0$ and $N \leq e^{|\log \varepsilon|^{1 / 4}}$ can ensure that $N_{0}\left(c_{1}, \rho, b, d\right) \leq N_{1} \leq|\log \varepsilon|^{\frac{1}{2 c_{1}}}$.

Proof. We will eliminate the variables $(\Theta, E) \in \mathbf{B}\left(100 b N^{2}\right) \times \mathcal{I}_{\varepsilon}$. This needs make quantitative restrictions on $\vec{\omega}$.

Let

$$
N^{c_{3}}<L<N^{c_{4}}
$$

where $0<c_{3}<c_{4}<1$ will be specified later. Let $S_{L}$ be the set of all $(\vec{\omega}, \Theta, E) \in$ $[0,2 \pi]^{b} \times \mathbf{B}\left(100 b N^{2}\right) \times \mathcal{I}_{\varepsilon}$ such that, for any $\mathbf{n} \in[-L, L]^{b}$ and $\vec{\omega} \in[0,2 \pi]^{b}, \Theta+\mathbf{n} \vec{\omega} \in$ $\mathbf{X}_{N_{2}}(E, \vec{\omega})$. Applying Proposition 3.3 gives

$$
\begin{aligned}
& \operatorname{deg}\left(S_{L}\right) \leq L^{C(b, d)}, \\
& \sup _{1 \leq j \leq d, \Theta_{j}^{\urcorner} \in \mathbb{R}^{d-1}} \operatorname{mes}\left(\mathbb{R} \backslash S_{L}\left(\Theta_{j}^{\urcorner}\right)\right) \leq e^{-N_{1}^{c_{1}} / 2} .
\end{aligned}
$$

Fix $I \subset\{1, \cdots, d\}$ and define $\mathcal{A}$ to be all $(\vec{\omega}, \Theta, y, E) \in[0,2 \pi]^{b} \times \mathbf{B}\left(100 b N^{2}\right) \times \mathbb{R}^{I} \times \mathcal{I}_{\varepsilon}$ satisfying

$$
\left(\vec{\omega},\left(\Theta_{j}+y_{j}\right)_{j \in I},\left(\Theta_{j}\right)_{j \notin I}, E\right) \notin S_{L} .
$$

Obviously, by (3.7) and (3.9),

$$
\operatorname{deg}(\mathcal{A}) \leq L^{C(b, d)}
$$

Fix $\vec{\omega} \in[0,2 \pi]^{b}$ and consider

$$
\mathcal{A}_{1}:=\mathcal{A}(\vec{\omega}) \subset \mathbf{B}\left(100 b N^{2}\right) \times \mathbb{R}^{I} \times \mathcal{I}_{\varepsilon} .
$$


Assume for $y_{I}=\left(y_{i}\right)_{i \in I}, y_{I} \notin \mathbf{B}_{I}\left(200 b N^{2}\right)=\left\{y \in \mathbb{R}^{I}:|y| \leq 200 b N^{2}\right\}$. Then for all $\mathbf{n} \in[-L, L]^{b}+\mathcal{E}_{N_{1}}^{0}$, one has since $|\Theta| \leq 100 b N^{2}$ and $|E| \leq|\log \varepsilon|^{\frac{1}{2 c_{1}}} \leq N$,

$$
\begin{aligned}
\sum_{i=1}^{d}\left(\Theta_{i}+y_{i}+\mathbf{n}_{i} \cdot \vec{\omega}_{i}\right)^{2}-E & \geq \sum_{i \in I}\left(\Theta_{i}+y_{i}+\mathbf{n}_{i} \cdot \vec{\omega}_{i}\right)^{2}-E \\
& \geq N^{4},
\end{aligned}
$$

which shows that there is no resonances in this case. In other words, we must have

$$
\mathcal{A}_{1} \subset \mathbf{B}\left(100 b N^{2}\right) \times \mathbf{B}_{I}\left(200 b N^{2}\right) \times \mathcal{I}_{\varepsilon} .
$$

By (3.10) and Tarski-Seidenberg principle (i.e., Lemma 2.2), we obtain

$$
\operatorname{deg}\left(\mathcal{A}_{1}\right) \leq L^{C(b, d)}
$$

From (3.8), for all $(\Theta, E) \in \mathbf{B}\left(100 b N^{2}\right) \times \mathcal{I}_{\varepsilon}$, we have

$$
\operatorname{mes}_{I}\left(\mathcal{A}_{1}(\Theta, E)\right) \leq \eta:=e^{-N_{1}^{c_{1}} / 2} .
$$

At this stage, we need a lemma for eliminating multi-variable:

Lemma 3.5. Let $\mathcal{S} \subset\left[0,1000 b N^{2}\right]^{s+r}$ be a semi-algebraic set of degree $B$ and such that

$$
\operatorname{mes}_{s}(\mathcal{S}(y))<\eta \text { for } \forall y \in\left[0,1000 b N^{2}\right]^{r} \text { and } \log (B N) \ll \log \frac{1}{\eta} \text {. }
$$

Then the set

$$
\left\{\left(x_{1}, \cdots, x_{2^{r}}\right) \in\left[0,1000 b N^{2}\right]^{s 2^{r}}: \bigcap_{1 \leq i \leq 2^{r}} \mathcal{S}\left(x_{i}\right) \neq \emptyset\right\}
$$

is semi-algebraic of degree at most $B^{C}$ and measure at most

$$
N^{C} B^{C} \eta^{s^{-r} 2^{-r(r-1) / 2}}
$$

where $C=C(s, r, b)>0$.

Proof of Lemma 3.5. The degree bound follows from Tarski-Seidenberg principle Lemma 2.2. The measure bound is derived as follows. We first divide $\left[0,1000 b N^{2}\right]^{s+r}$ into about $N^{C(s, r, b)}$ many unit cubes. Then applying Lemma 1.18 of [Bou07] gives measure bound $B^{C} \eta^{s^{-r} 2^{-r(r-1) / 2}}$ on each such unit cube. Finally, it suffices to take account of all those cubes.

From (3.13) and (3.14), we have by using Lemma 3.5 (with $s=|I|, r=d+1, B=$ $L^{C}$ and $\left.\eta=e^{-N_{1}^{c_{1}} / 2}\right)$ that

$$
\begin{gathered}
\mathcal{A}_{2}=\left\{\left(y^{(i)}\right)_{1 \leq i \leq 2^{d+1}}: \bigcap_{1 \leq i \leq 2^{d+1}} \mathcal{A}_{1}\left(y^{(i)}\right) \neq \emptyset\right\} \text { is a semi-algebraic set with } \\
\operatorname{deg}\left(\mathcal{A}_{2}\right) \leq L^{C(b, d,|I|)}, \operatorname{mes}\left(\mathcal{A}_{2}\right) \leq \eta_{1}:=N^{C(b, d,|I|)} \eta^{|I|^{-d-1} 2^{-d(d+1) / 2}} .
\end{gathered}
$$

Define

$$
\begin{aligned}
\mathcal{B} & =\left\{\left(\vec{\omega},\left(y^{(i)}\right)_{1 \leq i \leq 2^{d+1}}\right): \vec{\omega} \in[0,2 \pi]^{b}, \bigcap_{1 \leq i \leq 2^{d+1}} \mathcal{A}\left(\vec{\omega}, y^{(i)}\right) \neq \emptyset\right\} \\
& \subset[0,2 \pi]^{b} \times\left[0,200 b N^{2}\right]^{|I| 2^{d+1}} .
\end{aligned}
$$


By Lemma $2.2, \operatorname{deg}(\mathcal{B}) \leq L^{C(d,|I|)}$. Write $\bar{\omega}=\left(\vec{\omega}_{i}\right)_{i \notin I}$ and $\widetilde{\omega}:=\left(\vec{\omega}_{i}\right)_{i \in I}$. Then by Fubini Theorem and (3.15),

$$
\operatorname{mes}(\mathcal{B}(\bar{\omega})) \leq \eta_{1}, \operatorname{deg}(\mathcal{B}(\bar{\omega})) \leq L^{C}
$$

Notice that for any $C_{\star}>1$, one has $\frac{1}{\eta_{1}} \gg N^{C}$ if $N \geq N_{0}\left(C_{\star}, c_{1}, b, d,|I|\right)>0$. One considers the set $\mathcal{B}_{1}$ containing $\widetilde{\omega}$, which is defined by the following: there is some sequence $\mathbf{n}^{(1)}, \cdots, \mathbf{n}^{\left(2^{d+1}\right)} \in \mathbb{Z}^{b_{I}}\left(b_{I}=\sum_{i \in I} b_{i}\right)$ satisfying

$$
L^{\underline{C}_{\alpha}} \leq \min _{i \in I, 1 \leq j \leq b_{i}}\left|n_{i j}^{(\alpha)}\right| \leq\left|\mathbf{n}^{(\alpha)}\right| \leq L^{\bar{C}_{\alpha}}\left(1 \leq \alpha \leq 2^{d+1}\right),
$$

such that

where

$$
\left(\widetilde{\omega}, \mathbf{n}^{(1)} \widetilde{\omega}, \cdots, \mathbf{n}^{\left(2^{d+1}\right)} \widetilde{\omega}\right) \in \mathcal{B}(\bar{\omega}),
$$

$$
1 \ll \underline{C}_{1} \ll \bar{C}_{1} \ll \cdots \ll \underline{C}_{2^{d+1}} \ll \bar{C}_{2^{d+1}}
$$

At this stage, we will introduce a key lemma for eliminating frequencies:

Lemma 3.6. Let $\mathcal{S} \subset \mathbb{R}^{s r}$ be a semi-algebraic set of degree $B$ and $\operatorname{mes}(\mathcal{S})<\eta$ with $\eta>0$.

Let $b(r)=\sum_{i=1}^{r} b_{i}, b_{i} \in \mathbb{N}$. For $\vec{\omega}_{i} \in[0,2 \pi]^{b_{i}}, \vec{\omega}=\left(\vec{\omega}_{1}, \cdots, \vec{\omega}_{r}\right) \in[0,2 \pi]^{b(r)}$ and $\mathbf{n}=\left(\mathbf{n}_{1}, \cdots, \mathbf{n}_{r}\right) \in \mathbb{Z}^{b(r)}\left(\mathbf{n}_{i} \in \mathbb{Z}^{b_{i}}\right)$, define

$$
\mathbf{n} \vec{\omega}=\left(\mathbf{n}_{1} \cdot \vec{\omega}_{1}, \cdots, \mathbf{n}_{r} \cdot \vec{\omega}_{r}\right) .
$$

For any $C>1$, define $\mathcal{N}_{1}, \cdots, \mathcal{N}_{s-1} \subset \mathbb{Z}^{b(r)}$ to be finite sets with the following property:

$$
\min _{1 \leq i \leq r, 1 \leq j \leq b_{i}}\left|n_{i j}\right|>\left(B \max _{1 \leq i \leq r}\left|\mathbf{m}_{i}\right|\right)^{C}
$$

where $\mathbf{n} \in \mathcal{N}_{l}, \mathbf{m} \in \mathcal{N}_{l-1}(2 \leq l \leq s-1)$.

Then there is some $C=C(r, s, b)>0$ such that for $\max _{\mathbf{n} \in \mathcal{N}_{s-1}}|\mathbf{n}|^{C}<\frac{1}{\eta}$, one has

$$
\begin{aligned}
\operatorname{mes} & \left(\left\{\vec{\omega} \in[0,2 \pi]^{b(r)}: \exists \mathbf{n}^{(i)} \in \mathcal{N}_{i} \text { s.t., }\left(\vec{\omega}, \mathbf{n}^{(1)} \vec{\omega}, \cdots, \mathbf{n}^{(s-1)} \vec{\omega}\right) \in \mathcal{S}\right\}\right) \\
& \leq B^{C}\left(\min _{\mathbf{n} \in \mathcal{N}_{1}} \min _{i, j}\left|n_{i j}\right|\right)^{-1} .
\end{aligned}
$$

Proof of Lemma 3.6. The proof is similar to that of Lemma 1.20 in [Bou07] by iterating Lemma 2.3. The main difference is that we allow $\mathcal{S}$ to vary in $\mathbb{R}^{s r}$ rather than in the unit cube. Bourgain's proof remains applicable since Lemma 2.3 permits re-scaling. Moreover, at $i$-th $(i \leq s-1)$ iteration step, the valid sets are essentially contained in $\left[0, \max _{\mathbf{n} \in \mathcal{N}_{s-i}}|\mathbf{n}|\right]^{r(s-i+1)}$. We omit the details here.

Then from (3.16) and Lemma 3.6 (set $r=|I|, s=2^{d+1}+1$ ), we have

$$
\operatorname{mes}\left(\mathcal{B}_{1}\right) \leq L^{-\underline{C}_{1}} L^{C} \leq L^{-3} \text {. }
$$

Define

$$
\mathcal{G}_{L}:=\bigcap_{\emptyset \neq I \subset\{1, \cdots, d\}}\left\{\vec{\omega} \in[0,2 \pi]^{b}: \vec{\omega}=(\bar{\omega}, \widetilde{\omega}), \widetilde{\omega} \notin \mathcal{B}_{1}\right\} .
$$

Thus $\vec{\omega} \notin \mathcal{G}_{L}$ if and only if, there are $\emptyset \neq I \subset\{1, \cdots, d\}$ and some sequence $\mathbf{n}^{(1)}, \cdots, \mathbf{n}^{\left(2^{d+1}\right)} \in \mathbb{Z}^{b_{I}}$ satisfying (3.17) such that,

$$
\left(\vec{\omega}, \mathbf{n}^{(1)} \widetilde{\omega}, \cdots, \mathbf{n}^{\left(2^{d+1}\right)} \widetilde{\omega}\right) \in \mathcal{B} .
$$


This implies that $\mathcal{G}_{L}$ is a semi-algebraic set. Furthermore, by (3.17) and (3.18),

$$
\operatorname{mes}\left([0,2 \pi]^{b} \backslash \mathcal{G}_{L}\right) \leq L^{-2}, \operatorname{deg}\left(\mathcal{G}_{L}\right) \leq L^{C \bar{C}_{2 d+1}} .
$$

We should remark that $\mathcal{G}_{L}$ also depends on $\underline{C}_{1}, \cdots, \bar{C}_{2^{d+1}}$.

Finally, for $0<c_{3}(b, d) \ll c_{4}(b, d) \ll 1$, we can choose appropriate $L_{l}, \underline{C}_{1}, \cdots, \bar{C}_{2^{d+1}}$ and then iterate along every axis direction (i.e., induction on $I$ ) as done by Bourgain [Bou07] (see the proof of the ClAIM) if $\vec{\omega} \in \mathcal{G}_{L_{l}}$. The number of all possible $L_{l}, \underline{C}_{1}, \cdots, \bar{C}_{2^{d+1}}$ is finite and depends only on $b, d$. In particular, we have

$$
\begin{aligned}
\operatorname{deg}\left(\Omega_{N}\right) \leq C N^{c_{4} C \bar{C}_{2^{d+1}}} \leq N^{4 d}, \\
\operatorname{mes}\left([0,2 \pi]^{b} \backslash \Omega_{N}\right) \leq C N^{-2 c_{3}} \leq N^{-c_{2}},
\end{aligned}
$$

where $\Omega_{N}=\bigcap_{L_{l}, \underline{C}_{1}, \cdots, \bar{C}_{2^{d+1}}} \mathcal{G}_{L_{l}}$ and $0<c_{2}(b, d) \ll c_{3}(b, d)$. In addition, if $\vec{\omega} \in \Omega_{N}$, then for all $(E, \Theta) \in \mathcal{I}_{\varepsilon} \times \mathbf{B}\left(100 b N^{2}\right)$ there is some $\frac{1}{10} N^{c_{3}}<M<10 N^{c_{4}}$ such that for all $\mathbf{k} \in[-M, M]^{b} \backslash\left[-M^{\frac{1}{10 b}}, M^{\frac{1}{10 b}}\right]^{b}$, one has $\Theta+\mathbf{k} \vec{\omega} \notin \mathbf{X}_{N_{1}}$.

Combining the above proposition and Cartan's estimate on subharmonic functions, we can finish the proof of LDT in $\left[N, N^{2}\right]$.

Since (3.6) and (3.11), it suffices to consider in case

$$
\Theta \in \mathbf{B}\left(1000 b N^{2}\right) \text {. }
$$

Proposition 3.7. Assume the assumptions of Proposition 3.4 are satisfied. Let $\Theta$ satisfy (3.19). Then for $\vec{\omega} \in \Omega_{N}$, we have

(1) Fix $1 \leq j \leq d$ and $\Theta_{j}^{\urcorner} \in \mathbf{B}_{d-1}\left(1000 b N^{2}\right)$. Write $\Theta=\left(\Theta_{j}, \Theta_{j}^{\urcorner}\right) \in \mathbb{R}^{d}$. Assume there exist $\widetilde{N} \in\left[N^{c_{3}} / 4, N^{c_{4}}\right]$ and $\bar{\Lambda} \subset \Lambda \in \mathcal{E}_{\widetilde{N}}$ with $\operatorname{diam}(\bar{\Lambda}) \leq$ $4 \widetilde{N}^{\frac{1}{10 b}}$ and $\Lambda \subset \mathbf{B}\left(1000 b N^{2}\right)$ such that, for any $\mathbf{k} \in \Lambda \backslash \bar{\Lambda}$, there exists some $\mathcal{E}_{N_{1}} \ni W \subset \Lambda \backslash \bar{\Lambda}$ such that $\operatorname{dist}(\mathbf{k}, \Lambda \backslash \bar{\Lambda} \backslash W) \geq N_{1} / 2$, and $\Theta+\mathbf{k} \vec{\omega} \notin \mathbf{X}_{N_{1}}$. Let

$$
Y_{\Theta}=\left\{y \in \mathbb{R}:\left|y-\Theta_{j}\right| \leq e^{-10 \rho N_{1}},\left|\Theta_{j}\right| \leq 1000 b N^{2},\left\|G_{\Lambda}\left(E ;\left(y, \Theta_{j}^{\urcorner}\right)\right)\right\| \geq e^{\sqrt{\widetilde{N}}}\right\} .
$$

Then

$$
\operatorname{mes}\left(Y_{\Theta}\right) \leq e^{-\widetilde{N}^{\frac{1}{3}}}
$$

(2) Fix $N_{\star} \in\left[N, N^{2}\right]$. If $E \in \mathcal{I}_{\varepsilon}$ and $0<c_{1}<c_{3} / 10$, then there exists some set $\mathbf{X}_{N_{\star}}=\mathbf{X}_{N_{\star}}(E, \vec{\omega}) \subset \mathbb{R}^{d}$ such that

$$
\sup _{1 \leq j \leq d, \Theta_{j}^{\neg} \in \mathbb{R}^{d-1}} \operatorname{mes}\left(\mathbf{X}_{N_{\star}}\left(\Theta_{j}^{\urcorner}\right)\right) \leq e^{-N_{\star}^{c_{1}}}
$$

and for $\Theta \notin \mathbf{X}_{N_{\star}}, Q \in \mathcal{E}_{N_{\star}}^{0}$,

$$
\left|G_{Q}(E ; \Theta)\left(\mathbf{n}, \mathbf{n}^{\prime}\right)\right| \leq e^{-\left(\frac{4}{5} \rho-\frac{C(\rho, b, d)}{\sqrt{N_{1}}}\right)\left|\mathbf{n}-\mathbf{n}^{\prime}\right|} \text { for }\left|\mathbf{n}-\mathbf{n}^{\prime}\right| \geq N_{\star} / 10 .
$$

Proof. (1) Let $\mathcal{D}$ be the $e^{-10 \rho N_{1}}$ neighbourhood of $\Theta_{j}$ in the complex plane, i.e.,

$$
\mathcal{D}=\left\{y \in \mathbb{C}:|\Im y| \leq e^{-10 \rho N_{1}},\left|\Re y-\Theta_{j}\right| \leq e^{-10 \rho N_{1}}\right\} .
$$


From assumptions, we have for all $\mathbf{k} \in \Lambda \backslash \bar{\Lambda}$ and $Q \in \mathcal{E}_{N_{1}}^{0}$,

$$
\begin{aligned}
\left\|G_{Q}(E ; \Theta+\mathbf{k} \vec{\omega})\right\| & \leq e^{\sqrt{N_{1}}}, \\
\left|G_{Q}(E ; \Theta+\mathbf{k} \vec{\omega})\left(\mathbf{n}, \mathbf{n}^{\prime}\right)\right| & \leq e^{-\frac{4 \rho}{5}\left|\mathbf{n}-\mathbf{n}^{\prime}\right|} \text { for }\left|\mathbf{n}-\mathbf{n}^{\prime}\right| \geq N_{1} / 10 .
\end{aligned}
$$

Note that for all $\mathbf{n}, \mathbf{n}^{\prime} \in\left[-N_{1}, N_{1}\right]^{b}$,

$$
e^{-10 \rho N_{1}}<e^{-3 \rho N_{1}-\rho\left|\mathbf{n}-\mathbf{n}^{\prime}\right|} .
$$

Then by Lemma B.1, (3.20) and (3.21), we have for any $y \in \mathcal{D}, Q \in \mathcal{E}_{N_{1}}^{0}$ and $\mathbf{k} \in \Lambda \backslash \bar{\Lambda}$,

$$
\begin{aligned}
\left\|G_{Q}\left(E ;\left(\Theta_{j}+y, \Theta_{j}^{\urcorner}\right)+\mathbf{k} \vec{\omega}\right)\right\| & \leq 2 e^{\sqrt{N_{1}}} \\
\left|G_{Q}\left(E ;\left(\Theta_{j}+y, \Theta_{j}^{\urcorner}\right)+\mathbf{k} \vec{\omega}\right)\left(\mathbf{n}, \mathbf{n}^{\prime}\right)\right| & \leq 2 e^{-\frac{4 \rho}{5}\left|\mathbf{n}-\mathbf{n}^{\prime}\right|} \text { for }\left|\mathbf{n}-\mathbf{n}^{\prime}\right| \geq N_{1} / 10 .
\end{aligned}
$$

Applying Lemma B.2 with $M_{1}=M_{0}=N_{1}$ implies for any $y \in \mathcal{D}$,

$$
\left\|G_{\Lambda \backslash \bar{\Lambda}}\left(E ;\left(\Theta_{j}+y, \Theta_{j}^{\urcorner}\right)\right)\right\| \leq 4\left(2 N_{1}+1\right)^{b} e^{\sqrt{N_{1}}} \leq e^{2 \sqrt{N_{1}}} .
$$

We then can use the following matrix-valued Cartan's estimate to propagate the "smallness of measure":

Lemma 3.8 (Cartan's estimate, [Bou05]). Let $T(\theta)$ be a self-adjoint $N \times N$ matrix function of a parameter $\theta \in[-\delta, \delta]$ satisfying the following conditions:

(i) $T(\theta)$ is real analytic in $\theta \in[-\delta, \delta]$ and has a holomorphic extension to

$$
\mathcal{D}_{\delta, \delta_{1}}=\left\{\theta \in \mathbb{C}:|\Re \theta| \leq \delta,|\Im \theta| \leq \delta_{1}\right\}
$$

satisfying $\sup _{\theta \in \mathcal{D}_{\delta, \delta}}\|T(\theta)\| \leq K_{1}, K_{1} \geq 1$.

(ii) For all $\theta \in[-\delta, \delta]$, there is subset $V \subset[1, N]$ with $|V| \leq M$ such that

$$
\left\|\left(R_{[1, N] \backslash V} T(\theta) R_{[1, N] \backslash V}\right)^{-1}\right\| \leq K_{2}, K_{2} \geq 1 .
$$

(iii) Assume

$$
\operatorname{mes}\left\{\theta \in[-\delta, \delta]:\left\|T^{-1}(\theta)\right\| \geq K_{3}\right\} \leq 10^{-3} \delta\left(1+K_{1}\right)^{-1}\left(1+K_{2}\right)^{-1} .
$$

Let $0<\epsilon \leq\left(1+K_{1}+K_{2}\right)^{-10 M}$. Then

$$
\operatorname{mes}\left\{\theta \in[-\delta / 2, \delta / 2]:\left\|T^{-1}(\theta)\right\| \geq \epsilon^{-1}\right\} \leq C \delta e^{-\frac{c \log \epsilon^{-1}}{M \log \left(K_{1}+K_{2}+K_{3}\right)}},
$$

where $C, c>0$ are some absolute constants.

We will apply Lemma 3.8 with

$$
T(y)=h_{\Lambda}\left(\left(\Theta_{j}+y, \Theta_{j}^{\urcorner}\right)\right)-E, \delta=\delta_{1}=2 e^{-10 \rho N_{1}} .
$$

It suffices to verify the assumptions of Lemma 3.8. By assumptions (3.19), $\Lambda \subset$ $\mathbf{B}\left(1000 b N^{2}\right)$ and (3.24), one has

$$
K_{1}=O\left(N^{2}\right), M=|\bar{\Lambda}| \leq C(b, d) \tilde{N}^{1 / 10}, K_{2}=e^{2 \sqrt{N_{1}}} .
$$

Since LDT holds at scale $N_{2}$ for $y$ being outside a set of measure at most $e^{-N_{2}^{c_{1}}}$. Applying Lemma B.2 yields

$$
\left\|T^{-1}(y)\right\| \leq 4\left(2 N_{2}+1\right)^{b} e^{\sqrt{N_{2}}} \leq e^{2 \sqrt{N_{2}}}=K_{3}
$$

for $y$ being outside a set of measure at most

$$
(2 \tilde{N}+1)^{b} e^{-N_{2}^{c_{1}}} \leq e^{-N_{2}^{c_{1}} / 2} .
$$


It follows from $100 N_{1}<N_{1}^{3 / 2}<N_{2}^{c_{1}}$ that

$$
10^{-3} \delta_{1}\left(1+K_{1}\right)^{-1}\left(1+K_{2}\right)^{-1} \geq e^{-N_{2}^{c_{1}} / 2} .
$$

This verifies (iii) of Lemma 3.8. For $\epsilon=e^{-\sqrt{\widetilde{N}}}$, one has by (3.26) and (3.27), $\epsilon<\left(1+K_{1}+K_{2}\right)^{-10 M}$. By (3.25) of Lemma 3.8,

$$
\operatorname{mes}\left(Y_{\Theta}\right) \leq e^{-\frac{c \sqrt{\widehat{N}}}{N_{2} \widetilde{N}^{1 / 10} \log \widetilde{N}}} \leq e^{-\widetilde{N}^{1 / 3}}
$$

(2) Fix $N_{\star} \in\left[N, N^{2}\right]$ and $E \in \mathcal{I}_{\varepsilon}$. As done in (3.11), to prove LDT at scale $N_{\star}$ it suffices to restrict $\Theta \in \mathbf{B}\left(10 b N_{\star}\right) \subset \mathbf{B}\left(10 b N^{2}\right)$.

Fix $1 \leq j \leq d, \Theta_{j}^{\urcorner} \in \mathbb{R}^{d-1}$ and $\Theta=\left(\Theta_{j}, \Theta_{j}^{\urcorner}\right) \in \mathbf{B}\left(10 b N_{\star}\right)$. Then $\Theta+\mathbf{n} \vec{\omega} \in$ $\mathbf{B}\left(100 b N^{2}\right)$ for all $|\mathbf{n}| \leq N_{\star}$. By using Proposition 3.4 ( with $\Theta$ replaced by $\Theta+\mathbf{n} \vec{\omega}$ ), for such $\Theta$ and any $\mathbf{n} \in Q \in \mathcal{E}_{N_{\star}}^{0}$, there exist $\frac{1}{4} N^{c_{3}} \leq \widetilde{N} \leq N^{c_{4}}, \Lambda \in \mathcal{E}_{\widetilde{N}}$ and $\bar{\Lambda}$, such that

$$
\mathbf{n} \in \bar{\Lambda} \subset \Lambda \subset Q, \operatorname{dist}(\mathbf{n}, Q \backslash \Lambda) \geq \widetilde{N} / 2, \operatorname{diam}(\bar{\Lambda}) \leq 4 \widetilde{N}^{\frac{1}{10 b}} .
$$

Moreover, for any $\mathbf{k} \in \Lambda \backslash \bar{\Lambda}, \Theta+\mathbf{k} \vec{\omega} \notin \mathbf{X}_{N_{1}}$ and there exists some $\mathcal{E}_{N_{1}} \ni W \subset \Lambda \backslash \bar{\Lambda}$ such that $\mathbf{k} \in W, \operatorname{dist}(\mathbf{k}, \Lambda \backslash \bar{\Lambda} \backslash W) \geq N_{1} / 2$. We should remark that in the above argument we had applied essentially methods of [JLS20] in dealing with elementary regions (of size $\widetilde{N}$ ) near the boundary of $Q$.

We now fix above $\tilde{N}, \bar{\Lambda}, \Lambda$ throughout the set $\left\{\left(y, \Theta_{j}^{\urcorner}\right) \in \mathbb{R}^{d}:\left|y-\Theta_{j}\right| \leq\right.$ $\left.e^{-10 \rho N_{1}}\right\}$. Recalling Lemma B.1 and the above constructions, we have by (1) of Proposition 3.7 that there exists a $Y \subset\left\{y \in \mathbb{R}:\left|y-\Theta_{j}\right| \leq e^{-10 \rho N_{1}}\right\}$ such that

$$
\operatorname{mes}(Y) \leq e^{-\widetilde{N}^{1 / 3}},
$$

and for $\Theta_{j} \notin Y,\left\|G_{\Lambda}(E ; \Theta)\right\| \leq e^{\sqrt{\widetilde{N}}}$. Applying Lemma B.3 yields

$$
\left|G_{\Lambda}(E ; \Theta)\left(\mathbf{n}, \mathbf{n}^{\prime}\right)\right| \leq e^{-\left(\frac{4 \rho}{5}-\frac{C}{\sqrt{N_{1}}}\right)\left|\mathbf{n}-\mathbf{n}^{\prime}\right|} \text { for }\left|\mathbf{n}-\mathbf{n}^{\prime}\right| \geq \tilde{N} / 10 .
$$

Cover $\left[0,10 b N_{\star}\right]$ by pairwise disjoint $e^{-10 \rho N_{1}}$-size intervals and let

$$
\mathbf{X}_{N_{\star}}\left(\Theta_{j}^{\urcorner}\right)=\bigcup_{Q \in \mathcal{E}_{N_{\star}}^{0}, \mathbf{n} \in Q, \Theta=\left(\Theta_{j}, \Theta_{j}^{-}\right)} Y .
$$

We remark that while $\Theta=\left(\Theta_{j}, \Theta_{j}^{\urcorner}\right)$varies on a line for fixed $\Theta_{j}^{\urcorner}$, the total number of $Y$ is bounded by $10 N_{\star} e^{10 \rho N_{1}}$. Thus by (3.28), (3.29) and $c_{1}<c_{3} / 10$, one has

$$
\operatorname{mes}\left(\mathbf{X}_{N_{\star}}\left(\Theta_{j}^{\urcorner}\right)\right) \leq C\left(2 N_{\star}+1\right)^{b} e^{10 \rho N_{1}} e^{-\widetilde{N}^{1 / 3}} \leq e^{-N_{\star} c_{3} / 7} \leq e^{-N_{\star}{ }^{c_{1}}} .
$$

Suppose now $\Theta \notin \mathbf{X}_{N_{\star}}$. Applying Lemma B.2 yields

$$
\left\|G_{Q}(E ; \Theta)\right\| \leq 4\left(2 N^{c_{4}}+1\right)^{b} e^{\sqrt{N^{c_{4}}}} \leq e^{\sqrt{N_{\star}}} .
$$

Recalling Lemma B.3, we obtain

$$
\left|G_{Q}(E ; \Theta)\left(\mathbf{n}, \mathbf{n}^{\prime}\right)\right| \leq e^{-\left(\frac{4 \rho}{5}-\frac{C(\rho, b, d)}{\sqrt{N_{1}}}\right)\left|\mathbf{n}-\mathbf{n}^{\prime}\right|} \text { for }\left|\mathbf{n}-\mathbf{n}^{\prime}\right| \geq N_{\star} / 10 .
$$

\section{STEP 3: The General Step}

The proof is based on similar arguments as in STEP 2 . 
We define for $N \geq e^{|\log \varepsilon|^{1 / 4}}$ the following scales

$$
N_{1} \sim(\log N)^{2 / c_{1}}, N_{2} \sim N_{1}^{2 / c_{1}} .
$$

Then we have

Proposition 3.9. Let $\Omega_{N_{i}}(i=1,2)$ be semi-algebraic set satisfying $\operatorname{deg}\left(\Omega_{N_{i}}\right) \leq$ $N_{i}^{4 d}$ and let $\bar{\rho}_{i} \in[\rho / 2, \rho)$. Assume further the following holds: If $\vec{\omega} \in \Omega_{N_{i}}$ and $E \in$ $\mathcal{I}_{\varepsilon}$, then there exists some semi-algebraic set $\mathbf{X}_{N_{i}} \subset \mathbb{R}^{d}$ satisfying $\operatorname{deg}\left(\mathbf{X}_{N_{i}}\right) \leq N_{i}^{C}$ such that

$$
\sup _{1 \leq j \leq d, \Theta_{j}^{\neg} \in \mathbb{R}^{d-1}} \operatorname{mes}\left(\mathbf{X}_{N_{i}}\left(\Theta_{j}^{\urcorner}\right)\right) \leq e^{-N_{i}^{c_{1}}},
$$

and for $\Theta \notin \mathbf{X}_{N_{i}}, Q \in \mathcal{E}_{N_{i}}^{0}$,

$$
\begin{aligned}
\left\|G_{Q}(E ; \Theta)\right\| & \leq e^{\sqrt{N_{i}}} \\
\left|G_{Q}(E ; \Theta)\left(\mathbf{n}, \mathbf{n}^{\prime}\right)\right| & \leq e^{-\bar{\rho}_{i}\left|\mathbf{n}-\mathbf{n}^{\prime}\right|} \text { for }\left|\mathbf{n}-\mathbf{n}^{\prime}\right| \geq N_{i} / 10, \\
& (i=1,2) .
\end{aligned}
$$

Then exists some semi-algebraic set $\Omega_{N} \subset \Omega_{N_{1}} \cap \Omega_{N_{2}}$ with $\operatorname{deg}\left(\Omega_{N}\right) \leq N^{4 d}$ and $\operatorname{mes}\left(\left(\Omega_{N_{1}} \cap \Omega_{N_{2}}\right) \backslash \Omega_{N}\right) \leq N^{-c_{2}}$ such that, if $\vec{\omega} \in \Omega_{N}$, then for $N \geq N_{0}\left(c_{1}, \rho, b, d\right)>$ 0

(i) For all $E \in \mathcal{I}_{\varepsilon}$ and $\Theta \in \mathbf{B}\left(100 b N^{2}\right)$, there is $\frac{N^{c_{3}}}{10}<M<10 N^{c_{4}}$ such that for all $\mathbf{k} \in[-M, M]^{b} \backslash\left[-M^{\frac{1}{10 b}}, M^{\frac{1}{10 b}}\right]^{b}, \Theta+\mathbf{k} \vec{\omega} \notin \mathbf{X}_{N_{1}}$.

(ii) Fix $N_{\star} \in\left[N, N^{2}\right]$. Then there exists some set $\mathbf{X}_{N_{\star}}=\mathbf{X}_{N_{\star}}(E, \omega) \subset \mathbb{R}^{d}$ such that

$$
\sup _{1 \leq j \leq d, \Theta_{j}^{\urcorner} \in \mathbb{R}^{d-1}} \operatorname{mes}\left(\mathbf{X}_{N_{\star}}\left(\Theta_{j}^{\urcorner}\right)\right) \leq e^{-N_{\star}^{c_{1}}},
$$

and for $\Theta \notin \mathbf{X}_{N_{\star}}, Q \in \mathcal{E}_{N_{\star}}^{0}$,

$$
\begin{aligned}
\left\|G_{Q}(E ; \Theta)\right\| & \leq e^{\sqrt{N_{\star}}} \\
\left|G_{Q}(E ; \Theta)\left(\mathbf{n}, \mathbf{n}^{\prime}\right)\right| & \leq e^{-\left(\bar{\rho}_{1}-\frac{C(\rho, b, d)}{\sqrt{N_{1}}}\right)\left|\mathbf{n}-\mathbf{n}^{\prime}\right|} \text { for }\left|\mathbf{n}-\mathbf{n}^{\prime}\right| \geq N_{\star} / 10 .
\end{aligned}
$$

Proof. The proof is similar to that in STEP 2, and we omit the details here.

From the above arguments, we can propagate LDT from (scales)

$$
\left[N, N^{2 / c_{1}}\right]
$$

to

$$
\left[e^{N^{c_{1} / 2}}, e^{2 N^{c_{1} / 2}}\right] .
$$

Thus a standard MSA induction (on scales) can establish LDT on the whole interval $\left[N_{0}, \infty\right]$ (see [BGS02] or [JLS20] for details). This finishes the proof of Theorem 3.1 . 


\section{Proof of Theorem 1.1}

In this section we will prove Theorem 1.1 by using LDT and Aubry duality.

We begin with a useful lemma:

Lemma 4.1. Let $\Psi \in H^{2}\left(\mathbb{R}^{d}\right)$ satisfy

$$
(-\Delta+\varepsilon V(\vec{\theta}+x \vec{\omega})) \Psi(x)=E \Psi(x) .
$$

Let $\widehat{\Psi}$ be the Fourier transformation of $\Psi$. Then for a.e. $\Theta \in \mathbb{R}^{d}$ and any $\vec{\theta} \in \mathbb{T}^{b}$ the following holds: Let $Z=\left\{Z_{\mathbf{k}}\right\}_{\mathbf{k} \in \mathbb{Z}^{b}}$ satisfy $Z_{\mathbf{k}}=e^{-\mathbf{i k} \cdot \vec{\theta}} \widehat{\Psi}(\Theta+\mathbf{k} \vec{\omega})$. Then

$$
h(\Theta) Z=E Z,
$$

where $h(\Theta)$ is given by (2.1). Moreover, There is $C=C(\Theta, \Psi, b, d) \in(0, \infty)$ such that

$$
\left|Z_{\mathbf{k}}\right| \leq C(1+|\mathbf{k}|)^{5 b} \text { for } \forall \mathbf{k} \in \mathbb{Z}^{b} \text {. }
$$

Proof. It suffices to consider $\Psi \in C_{0}^{\infty}\left(\mathbb{R}^{d}\right)$. We note first (4.1) is equivalent to

$$
\|\xi\|^{2} \widehat{\Psi}(\xi)+\widehat{\varepsilon V \Psi}(\xi)=E \widehat{\Psi}(\xi),
$$

where $\|\xi\|^{2}=\sum_{i=1}^{d}\left|\xi_{i}\right|^{2}$. Since $V$ is quasi-periodic (and analytic), we have

$$
V(\vec{\theta}+x \vec{\omega})=\sum_{\mathbf{k} \in \mathbb{Z}^{b}} \widehat{V}_{\mathbf{k}} e^{\mathbf{i k} \cdot(\vec{\theta}+x \vec{\omega})}
$$

and as a result,

$$
\begin{aligned}
\widehat{V \Psi}(\xi) & =\int_{\mathbb{R}^{d}} V(\vec{\theta}+x \vec{\omega}) \Psi(x) e^{-\mathbf{i} x \cdot \xi} \mathrm{d} x \\
& =\sum_{\mathbf{m} \in \mathbb{Z}^{b}} \int_{\mathbb{R}^{d}} \widehat{V}_{\mathbf{m}} e^{\mathbf{i m} \cdot(\vec{\theta}+x \vec{\omega})} \Psi(x) e^{-\mathbf{i} x \cdot \xi} \mathrm{d} x \\
& =\sum_{\mathbf{m} \in \mathbb{Z}^{b}} \widehat{V}_{\mathbf{m}} e^{\mathbf{i} \mathbf{m} \cdot \vec{\theta}} \int_{\mathbb{R}^{d}} \Psi(x) e^{-\mathbf{i} x \cdot(\xi-\mathbf{m} \vec{\omega})} \mathrm{d} x \\
& =\sum_{\mathbf{m} \in \mathbb{Z}^{b}} \widehat{V}_{\mathbf{m}} e^{\mathbf{i} \mathbf{m} \cdot \vec{\theta}} \widehat{\Psi}(\xi-\mathbf{m} \vec{\omega}) .
\end{aligned}
$$

Combining (4.4) and (4.5) yields

$$
\|\xi\|^{2} \widehat{\Psi}(\xi)+\varepsilon \sum_{\mathbf{m} \in \mathbb{Z}^{b}} \widehat{V}_{m} e^{\mathbf{i m} \cdot \vec{\theta}} \widehat{\Psi}(\xi-\mathbf{m} \vec{\omega})=E \widehat{\Psi}(\xi) .
$$

Given $\mathbf{k} \in \mathbb{Z}^{b}$, we set $\xi=\Theta+\mathbf{k} \vec{\omega}$ in (4.6). Then

$$
\begin{aligned}
& e^{-i \mathbf{k} \cdot \vec{\theta}}\|\Theta+\mathbf{k} \vec{\omega}\|^{2} \widehat{\Psi}(\Theta+\mathbf{k} \vec{\omega})+\varepsilon \sum_{\mathbf{m} \in \mathbb{Z}^{b}} \widehat{V}_{\mathbf{k}-\mathbf{m}} e^{-\mathbf{i m} \cdot \vec{\theta}} \widehat{\Psi}(\Theta+\mathbf{m} \vec{\omega}) \\
& =E e^{-i \mathbf{k} \cdot \vec{\theta}} \widehat{\Psi}(\Theta+\mathbf{k} \vec{\omega}),
\end{aligned}
$$

which implies (4.2). 
We then deal with the polynomial bound (4.3). Since $\widehat{\Psi} \in L^{2}\left(\mathbb{R}^{d}\right)$, we have

$$
\begin{aligned}
\int_{\mathbb{R}^{d}} \sum_{\mathbf{k} \in \mathbb{Z}^{b}} \frac{\left|Z_{\mathbf{k}}\right|^{2}(\Theta)}{(1+|\mathbf{k}|)^{10 b}} \mathrm{~d} \Theta & =\sum_{\mathbf{k} \in \mathbb{Z}^{b}} \frac{1}{(1+|\mathbf{k}|)^{10 b}} \int_{\mathbb{R}^{d}}|\widehat{\Psi}(\Theta+\mathbf{k} \vec{\omega})|^{2} \mathrm{~d} \Theta \\
& =\|\widehat{\Psi}\|_{L^{2}}^{2} \sum_{\mathbf{k} \in \mathbb{Z}^{b}} \frac{1}{(1+|\mathbf{k}|)^{10 b}}<\infty,
\end{aligned}
$$

which implies for a.e. $\Theta \in \mathbb{R}^{d}$,

$$
\sum_{\mathbf{k} \in \mathbb{Z}^{b}} \frac{\left|Z_{\mathbf{k}}\right|^{2}(\Theta)}{(1+|\mathbf{k}|)^{10 b}}:=C(\Theta, \Psi, b, d)<\infty .
$$

This means that for a.e. $\Theta \in \mathbb{R}^{d}$,

$$
\left|Z_{\mathbf{k}}\right| \leq C(\Theta, \Psi, b, d)(1+|\mathbf{k}|)^{5 b} \text { for } \forall \mathbf{k} \in \mathbb{Z}^{b} .
$$

We then prove our main result Theorem 1.1

Proof of Theorem 1.1. Let

$$
\widetilde{\Theta}=\frac{\Theta}{K}, \widetilde{E}=\frac{E}{K^{2}}, \varepsilon=\frac{\lambda}{K^{2}} .
$$

Suppose now $H(\vec{\theta})$ admits some eigenvalue $E \in\left[-K^{2}\left(\log \frac{K^{2}}{\lambda}\right)^{\frac{1}{2 c_{1}}}, K^{2}\left(\log \frac{K^{2}}{\lambda}\right)^{\frac{1}{2 c_{1}}}\right]$, i.e., there is some $\Psi \in H^{2}\left(\mathbb{R}^{d}\right)$ such that $H(\vec{\theta}) \Psi=E \Psi, \Psi \neq 0$. Then we have $\widetilde{E} \in \mathcal{I}_{\varepsilon}$ (see (3.6)) and

$$
(-\Delta+\lambda V(\vec{\theta}+K x \vec{\omega})) \Psi(x)=E \Psi(x) .
$$

Using Lemma 4.1, we know $Z(\Theta)=\left\{Z_{\mathbf{k}}=e^{-\mathbf{k} \cdot \vec{\theta}} \widehat{\Psi}(\Theta+K \mathbf{k} \vec{\omega})\right\}_{\mathbf{k} \in \mathbb{Z}^{b}}$ satisfies

$$
\sum_{\mathbf{k}^{\prime} \in \mathbb{Z}^{b}} \lambda \widehat{V}_{\mathbf{k}-\mathbf{k}^{\prime}} Z_{\mathbf{k}^{\prime}}(\Theta)+\sum_{i=1}^{d}\left(\Theta_{i}+K \mathbf{k}_{i} \cdot \vec{\omega}_{i}\right)^{2} Z_{\mathbf{k}}(\Theta)=E Z_{\mathbf{k}}(\Theta) .
$$

Obviously, (4.7) is equivalent to

$$
\sum_{\mathbf{k}^{\prime} \in \mathbb{Z}^{b}} \frac{\lambda}{K^{2}} \widehat{V}_{\mathbf{k}-\mathbf{k}^{\prime}} Z_{\mathbf{k}^{\prime}}(\Theta)+\sum_{i=1}^{d}\left(\Theta_{i} / K+\mathbf{k}_{i} \cdot \vec{\omega}_{i}\right)^{2} Z_{\mathbf{k}}(\Theta)=\frac{E}{K^{2}} Z_{\mathbf{k}}(\Theta),
$$

or in the new coordinate $(\widetilde{\Theta}, \widetilde{E}, \varepsilon)$,

$$
\sum_{\mathbf{k}^{\prime} \in \mathbb{Z}^{b}} \varepsilon \widehat{V}_{\mathbf{k}-\mathbf{k}^{\prime}} \widetilde{Z}_{\mathbf{k}^{\prime}}(\widetilde{\Theta})+\sum_{i=1}^{d}\left(\widetilde{\Theta}_{i}+\mathbf{k}_{i} \cdot \vec{\omega}_{i}\right)^{2} \widetilde{Z}_{\mathbf{k}}(\widetilde{\Theta})=\widetilde{E} \widetilde{Z}_{\mathbf{k}}(\widetilde{\Theta}),
$$

where

$$
\widetilde{Z}_{\mathbf{k}}(\widetilde{\Theta}):=Z_{\mathbf{k}}(\Theta) \text { for } \forall \mathbf{k} \in \mathbb{Z}^{b} .
$$

Note that (4.8) has the form of $h(\widetilde{\Theta}) \widetilde{Z}=\widetilde{E} \widetilde{Z}$ with $h(\widetilde{\Theta})$ being given by (2.1). Furthermore, recalling (4.3) and (4.9), we have for a.e. $\widetilde{\Theta}$,

$$
\left|\widetilde{Z}_{\mathbf{k}}(\widetilde{\Theta})\right| \leq C(\widetilde{\Theta}, \Psi, b, d)(1+|\mathbf{k}|)^{5 b} .
$$


Let $\delta>0$ be given. We obtain by Theorem 3.1 that for $0<\varepsilon=\frac{\lambda}{K^{2}} \leq$ $\varepsilon_{0}\left(\delta, c_{1}, \rho, b, d\right)$,

$$
\operatorname{mes}\left([0,2 \pi]^{b} \backslash \bigcap_{N \geq N_{0}} \Omega_{N}\right) \leq \delta .
$$

We fix $\vec{\omega} \in \bigcap_{N \geq N_{0}} \Omega_{N}$. Then since $\widetilde{E} \in \mathcal{I}_{\varepsilon}$ and $N \geq N_{0}$, there exists some set $\mathbf{X}_{N}=\mathbf{X}_{N}(\vec{\omega}, \widetilde{E}) \subset \mathbb{R}^{d}$ such that

$$
\sup _{1 \leq j \leq d, \widetilde{\Theta}_{j}^{\neg} \in \mathbb{R}^{d-1}} \operatorname{mes}\left(\mathbf{X}_{N}\left(\widetilde{\Theta}_{j}^{\neg}\right)\right) \leq e^{-N^{c_{1}}}
$$

and for $\widetilde{\Theta} \notin \mathbf{X}_{N}, Q \in \mathcal{E}_{N}^{0}$,

$$
\begin{aligned}
\left\|G_{Q}(\widetilde{E} ; \widetilde{\Theta})\right\| & \leq e^{\sqrt{N}} \\
\left|G_{Q}(\widetilde{E} ; \widetilde{\Theta})\left(\mathbf{n}, \mathbf{n}^{\prime}\right)\right| & \leq e^{-\frac{\rho}{10}\left|\mathbf{n}-\mathbf{n}^{\prime}\right|} \text { for }\left|\mathbf{n}-\mathbf{n}^{\prime}\right| \geq N / 10 .
\end{aligned}
$$

We should remark that (4.12) and (4.13) obviously hold true if $|\widetilde{\Theta}| \geq 10 b N$. Without loss of generality, we may assume $\mathbf{X}_{N} \subset \mathbf{B}(10 b N)$. As a result, we have by Fubini Theorem and (4.11) that

$$
\operatorname{mes}\left(\mathbf{X}_{N}\right) \leq C N^{d-1} e^{-N^{c_{1}}} \leq e^{-N^{c_{1}} / 2}
$$

and

$$
\sum_{N \geq N_{0}} \operatorname{mes}\left(\mathbf{X}_{N}\right)<\infty
$$

Then we obtain by using Borel-Cantelli Theorem that

$$
\operatorname{mes}\left(\mathbf{X}_{\infty}\right)=0
$$

where

$$
\mathbf{X}_{\infty}=\bigcap_{N \geq N_{0}} \bigcup_{M \geq N} \mathbf{X}_{M}
$$

Assuming $\widetilde{\Theta} \notin \mathbf{X}_{\infty}$, then there exists $N_{1}(\widetilde{\Theta}) \geq N_{0}$ such that $\widetilde{\Theta} \notin \mathbf{X}_{N}$ for all $N \geq N_{1}$. Recall that the Poisson's identity: For $h(\widetilde{\Theta}) \widetilde{Z}=\widetilde{E} \widetilde{Z}$ and $\mathbf{n} \in \Lambda \subset \mathbb{Z}^{b}$,

$$
\widetilde{Z}_{\mathbf{n}}(\widetilde{\Theta})=-\varepsilon \sum_{\mathbf{n}^{\prime} \in \Lambda, \mathbf{n}^{\prime \prime} \notin \Lambda} G_{\Lambda}(\widetilde{E} ; \widetilde{\Theta})\left(\mathbf{n}, \mathbf{n}^{\prime}\right) \widehat{V}_{\mathbf{n}^{\prime}-\mathbf{n}^{\prime \prime}} \widetilde{Z}_{\mathbf{n}^{\prime \prime}}(\widetilde{\Theta}) .
$$

Thus for a.e. $\widetilde{\Theta}$ and $N \geq N_{1}$,

$$
\begin{aligned}
\left|\widetilde{Z}_{\mathbf{0}}(\widetilde{\Theta})\right| & =\left|\sum_{|\mathbf{n}| \leq N,\left|\mathbf{n}^{\prime}\right|>N} G_{[-N, N]^{b}}(\widetilde{E} ; \widetilde{\Theta})(\mathbf{0}, \mathbf{n}) \widehat{V}_{\mathbf{n}-\mathbf{n}^{\prime}} \widetilde{Z}_{\mathbf{n}^{\prime}}(\widetilde{\Theta})\right| \\
& \leq C(\widetilde{\Theta}, \Psi, b, d) \sum_{|\mathbf{n}| \leq N,\left|\mathbf{n}^{\prime}\right|>N} e^{-\frac{\rho}{10}|\mathbf{n}|+\frac{\rho N}{100}+\sqrt{N}} e^{-\rho\left|\mathbf{n}-\mathbf{n}^{\prime}\right|}\left|\mathbf{n}^{\prime}\right|^{5 b} \\
& \leq C(\widetilde{\Theta}, \Psi, b, d) N^{b} \sum_{\left|\mathbf{n}^{\prime}\right|>N} e^{-\frac{\rho}{10}\left|\mathbf{n}^{\prime}\right|+\frac{\rho N}{100}+\sqrt{N}}\left|\mathbf{n}^{\prime}\right|^{5 b} \\
& \leq e^{-\frac{\rho N}{20}}
\end{aligned}
$$


Letting $N \rightarrow \infty$ in $(4.14)$, we have $\widetilde{Z}_{\mathbf{0}}(\widetilde{\Theta})=0$ for a.e. $\widetilde{\Theta}$. This implies

$$
0=\int_{\mathbb{R}^{d}}\left|\widetilde{Z}_{\mathbf{0}}(\widetilde{\Theta})\right|^{2} \mathrm{~d} \widetilde{\Theta}=K^{-d} \int_{\mathbb{R}^{d}}|\widehat{\Psi}(\Theta)|^{2} \mathrm{~d} \Theta,
$$

and $\Psi=0$, a contradiction.

We have shown $H(\vec{\theta})$ has no eigenvalues in $\left[-K^{2}\left(\log \frac{K^{2}}{\lambda}\right)^{\frac{1}{2 c_{1}}}, K^{2}\left(\log \frac{K^{2}}{\lambda}\right)^{\frac{1}{2 c_{1}}}\right]$.

\section{Appendix A.}

Lemma A.1. Let $H=-\Delta+V(x)$ be a Schrödinger operator with $M=\sup _{x \in \mathbb{R}^{d}}|V(x)|<$ $\infty$. Then for any $E \geq 0$, we have

$$
\sigma(H) \cap[E-M, E+M] \neq \emptyset,
$$

where $\sigma(H)$ denotes the spectrum of $H$.

Proof. Let $\mathbb{E}(\cdot)$ be the (projection-valued) spectral measure of $H=-\Delta+V(x)$. Then the Spectral Theorem reads as

$$
H=\int_{\sigma(H)} \lambda \mathrm{d} \mathbb{E}(\lambda)
$$

Note that $\sigma(-\Delta)=\sigma_{\text {ess }}(-\Delta)=[0, \infty)$. From Weyl criterion, for each $E \geq 0$ there is a sequence $\left\{F_{n}(x)\right\}_{n \in \mathbb{N}} \subset H^{2}\left(\mathbb{R}^{d}\right)$ such that

$$
\begin{aligned}
& (-\Delta-E) F_{n} \rightarrow 0 \text { as } n \rightarrow \infty, \\
& \left\|F_{n}\right\|_{L^{2}}=1 \text { for } \forall n .
\end{aligned}
$$

For $n \in \mathbb{N}$, we know $\mu_{n}(\cdot)=\left\langle\mathbb{E}(\cdot) F_{n}, F_{n}\right\rangle$ is a positive Borel measure and $\mu_{n}(\sigma(H))=$ $\left\|F_{n}\right\|_{L^{2}}^{2}=1$, where $\langle F, G\rangle=\int_{\mathbb{R}^{d}} F(x) \bar{G}(x) \mathrm{d} x$. Obviously, $\left\|V F_{n}\right\|_{L^{2}} \leq M$. Hence

$$
\begin{aligned}
\inf _{\lambda \in \sigma(H)}|\lambda-E|^{2}\left\|F_{n}\right\|_{L^{2}}^{2} & \leq \int_{\sigma(H)}|\lambda-E|^{2} \mathrm{~d} \mu_{n}(\lambda) \\
& =\left\|(-\Delta+V-E) F_{n}\right\|_{L^{2}}^{2} \\
& \leq\left(\left\|(-\Delta-E) F_{n}\right\|_{L^{2}}+M\right)^{2} .
\end{aligned}
$$

Letting $n \rightarrow \infty$ in (A.1), we get

$$
\operatorname{dist}(E, \sigma(H)) \leq M,
$$

which shows $[E-M, E+M] \cap \sigma(H) \neq \emptyset$. For otherwise, there must be $\operatorname{dist}(E, \sigma(H)) \geq$ $2 M$, which contradicts with (A.2).

\section{Appendix B.}

We write $G_{(\cdot)}=G_{(\cdot)}(E ; \Theta)$ for simplicity.

We have the following lemmas:

Lemma B.1 (Lemma A.1, [Shi19a]). Fix $\bar{\rho}>0$. Let $\Lambda \subset \mathbb{Z}^{b}$ satisfy $\Lambda \in \mathcal{E}_{N}$ and let $A, B$ be two linear operators on $\mathbb{C}^{\Lambda}$. We assume further

$$
\begin{aligned}
\left\|A^{-1}\right\| & \leq e^{\sqrt{N}} \\
\left|A^{-1}\left(\mathbf{n}, \mathbf{n}^{\prime}\right)\right| & \leq e^{-\bar{\rho}\left|\mathbf{n}-\mathbf{n}^{\prime}\right|} \text { for }\left|\mathbf{n}-\mathbf{n}^{\prime}\right| \geq N / 10 .
\end{aligned}
$$


Suppose that for all $\mathbf{n}, \mathbf{n}^{\prime} \in \Lambda$,

$$
\left|(B-A)\left(\mathbf{n}, \mathbf{n}^{\prime}\right)\right| \leq e^{-3 \bar{\rho} N-\bar{\rho}\left|\mathbf{n}-\mathbf{n}^{\prime}\right|} .
$$

Then

$$
\begin{aligned}
\left\|B^{-1}\right\| & \leq 2\left\|A^{-1}\right\|, \\
\left|B^{-1}\left(\mathbf{n}, \mathbf{n}^{\prime}\right)\right| & \leq\left|A^{-1}\left(\mathbf{n}, \mathbf{n}^{\prime}\right)\right|+e^{-\bar{\rho}\left|\mathbf{n}-\mathbf{n}^{\prime}\right|} .
\end{aligned}
$$

Lemma B.2 (Lemma 3.2, [JLS20]). Let $\bar{\rho} \in(\epsilon, \rho], M_{1} \leq N$ and $\operatorname{diam}(\Lambda) \leq 2 N+1$. Suppose that for any $\mathbf{n} \in \Lambda$, there exists some $W=W(\mathbf{n}) \in \mathcal{E}_{M}$ with $M_{0} \leq M \leq$ $M_{1}$ such that $\mathbf{n} \in W \subset \Lambda$, $\operatorname{dist}(\mathbf{n}, \Lambda \backslash W) \geq M / 2$ and

$$
\begin{aligned}
\left\|G_{W}\right\| & \leq 2 e^{\sqrt{M}}, \\
\left|G_{W}\left(\mathbf{n}, \mathbf{n}^{\prime}\right)\right| & \leq 2 e^{-\bar{\rho}\left|n-n^{\prime}\right|} \text { for }\left|\mathbf{n}-\mathbf{n}^{\prime}\right| \geq M / 10 .
\end{aligned}
$$

We assume further that $M_{0} \geq M_{0}(\epsilon, \rho, b, d)>0$. Then

$$
\left\|G_{\Lambda}\right\| \leq 4\left(2 M_{1}+1\right)^{b} e^{\sqrt{M_{1}}} .
$$

Lemma B.3 (Theorem 3.3, [JLS20]). Let $\Lambda_{1} \subset \Lambda \subset \mathbb{Z}^{b}$ satisfy $\operatorname{diam}(\Lambda) \leq 2 N+1$, $\operatorname{diam}\left(\Lambda_{1}\right) \leq N^{\frac{1}{3 b}}$. Let $M_{0} \geq(\log N)^{2}$ and $\bar{\rho} \in\left[\frac{\rho}{2}, \rho\right]$. Suppose that for any $\mathbf{n} \in$ $\Lambda \backslash \Lambda_{1}$, there exists some $W=W(\mathbf{n}) \in \mathcal{E}_{M}$ with $M_{0} \leq M \leq N^{1 / 3}$ such that $\mathbf{n} \in W \subset \Lambda \backslash \Lambda_{1}, \operatorname{dist}\left(\mathbf{n}, \Lambda \backslash \Lambda_{1} \backslash W\right) \geq M / 2$ and

$$
\begin{aligned}
\left\|G_{W}\right\| & \leq e^{\sqrt{M}}, \\
\left|G_{W}\left(\mathbf{n}, \mathbf{n}^{\prime}\right)\right| & \leq e^{-\bar{\rho}\left|\mathbf{n}-\mathbf{n}^{\prime}\right|} \text { for }\left|\mathbf{n}-\mathbf{n}^{\prime}\right| \geq M / 10 .
\end{aligned}
$$

Suppose further that

$$
\left\|G_{\Lambda}\right\| \leq e^{\sqrt{N}}
$$

Then

$$
\left|G_{\Lambda}\left(\mathbf{n}, \mathbf{n}^{\prime}\right)\right| \leq e^{-\left(\bar{\rho}-\frac{C}{\sqrt{M_{0}}}\right)\left|\mathbf{n}-\mathbf{n}^{\prime}\right|} \text { for }\left|\mathbf{n}-\mathbf{n}^{\prime}\right| \geq N / 10,
$$

where $C=C(\rho, b, d)>0$ and $N \geq N_{0}(\rho, b, d)>0$.

\section{REFERENCES}

[Agm70] S. Agmon. Lower bounds for solutions of Schrödinger equations. J. Analyse Math., 23:1-25, 1970.

[BG00] J. Bourgain and M. Goldstein. On nonperturbative localization with quasi-periodic potential. Ann. of Math. (2), 152(3):835-879, 2000.

[BGS02] J. Bourgain, M. Goldstein, and W. Schlag. Anderson localization for Schrödinger operators on $\mathbf{Z}^{2}$ with quasi-periodic potential. Acta Math., 188(1):41-86, 2002.

[BJ02] J. Bourgain and S. Jitomirskaya. Absolutely continuous spectrum for 1D quasiperiodic operators. Invent. Math., 148(3):453-463, 2002.

[Bje06] K. Bjerklöv. Positive Lyapunov exponents for continuous quasiperiodic Schrödinger equations. J. Math. Phys., 47(2):022702, 4, 2006.

[Bje07] K. Bjerklöv. Positive Lyapunov exponent and minimality for the continuous 1- $d$ quasiperiodic Schrödinger equations with two basic frequencies. Ann. Henri Poincaré, 8(4):687-730, 2007.

[BK19] J. Bourgain and I. Kachkovskiy. Anderson localization for two interacting quasiperiodic particles. Geom. Funct. Anal., 29(1):3-43, 2019.

[BKV17] I. Binder, D. Kinzebulatov, and M. Voda. Non-perturbative localization with quasiperiodic potential in continuous time. Comm. Math. Phys., 351(3):1149-1175, 2017. 
[Bou05] J. Bourgain. Green's function estimates for lattice Schrödinger operators and applications, volume 158 of Annals of Mathematics Studies. Princeton University Press, Princeton, NJ, 2005.

[Bou07] J. Bourgain. Anderson localization for quasi-periodic lattice Schrödinger operators on $\mathbb{Z}^{d}, d$ arbitrary. Geom. Funct. Anal., 17(3):682-706, 2007.

[Dam17] D. Damanik. Schrödinger operators with dynamically defined potentials. Ergodic Theory Dynam. Systems, 37(6):1681-1764, 2017.

[DFG19] D. Damanik, J. Fillman, and A. Gorodetski. Multidimensional almost-periodic Schrödinger operators with Cantor spectrum. Ann. Henri Poincaré, 20(4):1393-1402, 2019.

[DG14] D. Damanik and M. Goldstein. On the inverse spectral problem for the quasi-periodic Schrödinger equation. Publ. Math. Inst. Hautes Études Sci., 119:217-401, 2014.

[DS75] E. I. Dinaburg and Y. Sinai. The one-dimensional Schrödinger equation with quasiperiodic potential. Funct. Anal. Appl., 9:279-289, 1975.

[Eli92] L. H. Eliasson. Floquet solutions for the 1-dimensional quasi-periodic Schrödinger equation. Comm. Math. Phys., 146(3):447-482, 1992.

[FH83] R. Froese and I. Herbst. Exponential bounds and absence of positive eigenvalues for N-body Schrödinger operators. Comm. Math. Phys., 87(3):429-447, 1982/83.

[FS83] J. Fröhlich and T. Spencer. Absence of diffusion in the Anderson tight binding model for large disorder or low energy. Comm. Math. Phys., 88(2):151-184, 1983.

[FSW90] J. Fröhlich, T. Spencer, and P. Wittwer. Localization for a class of one-dimensional quasi-periodic Schrödinger operators. Comm. Math. Phys., 132(1):5-25, 1990.

[GS08] M. Goldstein and W. Schlag. Fine properties of the integrated density of states and a quantitative separation property of the Dirichlet eigenvalues. Geom. Funct. Anal., 18(3):755-869, 2008.

[IJ03] A. D. Ionescu and D. Jerison. On the absence of positive eigenvalues of Schrödinger operators with rough potentials. Geom. Funct. Anal., 13(5):1029-1081, 2003.

[JK85] D. Jerison and C. E. Kenig. Unique continuation and absence of positive eigenvalues for Schrödinger operators. Ann. of Math. (2), 121(3):463-494, 1985. With an appendix by E. M. Stein.

[JK16] S. Jitomirskaya and I. Kachkovskiy. $L^{2}$-reducibility and localization for quasiperiodic operators. Math. Res. Lett., 23(2):431-444, 2016.

[JLS20] S. Jitomirskaya, W. Liu, and Y. Shi. Anderson localization for multi-frequency quasiperiodic operators on $\mathbb{Z}^{d}$. Geom. Funct. Anal., 30(2):457-481, 2020.

[Kat59] T. Kato. Growth properties of solutions of the reduced wave equation with a variable coefficient. Comm. Pure Appl. Math., 12:403-425, 1959.

[KLSS17] Y. Karpeshina, Y.-R. Lee, R. Shterenberg, and G. Stolz. Ballistic transport for the Schrödinger operator with limit-periodic or quasi-periodic potential in dimension two. Comm. Math. Phys., 354(1):85-113, 2017.

[KS13] Y. Karpeshina and R. Shterenberg. Multiscale analysis in momentum space for quasiperiodic potential in dimension two. J. Math. Phys., 54(7):073507, 92, 2013.

[KS19] Y. Karpeshina and R. Shterenberg. Extended states for the Schrödinger operator with quasi-periodic potential in dimension two. Mem. Amer. Math. Soc., 258(1239):v+139, 2019.

[Liu18] W. Liu. Continuous quasiperiodic Schrödinger operators with Gordon type potentials. J. Math. Phys., 59(6):063501, 6, 2018.

[Mar19] A. Martin. A new class of Schrödinger operators without positive eigenvalues. Integral Equations Operator Theory, 91(3):Paper No. 24, 34, 2019.

[MJ17] C. A. Marx and S. Jitomirskaya. Dynamics and spectral theory of quasi-periodic Schrödinger-type operators. Ergodic Theory Dynam. Systems, 37(8):2353-2393, 2017.

[MP84] J. Moser and J. Pöschel. An extension of a result by Dinaburg and Sinai on quasiperiodic potentials. Comment. Math. Helv., 59(1):39-85, 1984.

[Par08] L. Parnovski. Bethe-Sommerfeld conjecture. Ann. Henri Poincaré, 9(3):457-508, 2008.

[PS10] L. Parnovski and A. Sobolev. Bethe-Sommerfeld conjecture for periodic operators with strong perturbations. Invent. Math., 181(3):467-540, 2010.

[PS12] L. Parnovski and R. Shterenberg. Complete asymptotic expansion of the integrated density of states of multidimensional almost-periodic Schrödinger operators. Ann. of Math. (2), 176(2):1039-1096, 2012. 
[PS16] L. Parnovski and R. Shterenberg. Complete asymptotic expansion of the spectral function of multidimensional almost-periodic Schrödinger operators. Duke Math. J., 165(3):509-561, 2016.

[Rüs80] H. Rüssmann. On the one-dimensional Schrödinger equation with a quasiperiodic potential. Ann. New York Acad. Sci., 357:90-107, 1980.

[Shi19a] Y. Shi. Analytic solutions of nonlinear elliptic equations on rectangular tori. J. Differential Equations, 267(9):5576-5600, 2019.

[Shi19b] Y. Shi. Spectral theory of multi-frequency quasi-periodic operator with gevrey type perturbation. arXiv: 1909.08772, 2019.

[Sim69] B. Simon. On positive eigenvalues of one-body Schrödinger operators. Comm. Pure Appl. Math., 22:531-538, 1969.

[SS91] E. Sorets and T. Spencer. Positive Lyapunov exponents for Schrödinger operators with quasi-periodic potentials. Comm. Math. Phys., 142(3):543-566, 1991.

[Sur90] S. Surace. The Schrödinger equation with a quasi-periodic potential. Trans. Amer. Math. Soc., 320(1):321-370, 1990.

[YZ14] J. You and Q. Zhou. Phase transition and semi-global reducibility. Comm. Math. Phys., 330(3):1095-1113, 2014.

(Y. Shi) College of Mathematics, Sichuan University, Chengdu 610064, China 